\title{
Reporting of Perinatal Outcomes in Probiotic Randomized Controlled Trials. A Systematic Review and Meta-Analysis
}

\author{
Íñigo María Pérez-Castillo ${ }^{1}$, Rafael Fernández-Castillo ${ }^{2, *(1)}$, Agustín Lasserrot-Cuadrado ${ }^{1}$, \\ José Luís Gallo-Vallejo ${ }^{3}$, Ana María Rojas-Carvajal ${ }^{1}$ and María José Aguilar-Cordero ${ }^{1,2}$ \\ 1 Andalusian Research, Development and Innovation Plan, CTS 367, University of Granada, \\ 18001 Granada, Spain; perezcastillo@correo.ugr.es (Í.M.P.-C.); lasserrot@ugr.es (A.L.-C.); \\ Anamar.rojascarvajal@gmail.com (A.M.R.-C.); mariajaguilar@telefonica.net (M.J.A.-C.) \\ 2 Faculty of Health Sciences, University of Granada, 18071 Granada, Spain \\ 3 Obstetrics and Gynecology Service, Virgen de las Nieves University Hospital, 18014 Granada, Spain; \\ jgallov@gmail.com \\ * Correspondence: rafaelfernandez@ugr.es; Tel.: +34-657-84-17-51
}

Citation: Pérez-Castillo, Í.M.;

Fernández-Castillo, R.; Lasserrot-Cuadrado, A.; Gallo-Vallejo, J.L.; Rojas-Carvajal, A.M.; Aguilar-Cordero, M.J. Reporting of Perinatal Outcomes in Probiotic Randomized Controlled Trials. A Systematic Review and Meta-Analysis. Nutrients 2021, 13 256. https://doi.org/10.3390/nu 13010256

Received: 17 December 2020 Accepted: 15 January 2021 Published: 17 January 2021

Publisher's Note: MDPI stays neutral with regard to jurisdictional clai$\mathrm{ms}$ in published maps and institutional affiliations.

Copyright: $(\odot 2021$ by the authors. Licensee MDPI, Basel, Switzerland. This article is an open access article distributed under the terms and conditions of the Creative Commons Attribution (CC BY) license (https:// creativecommons.org/licenses/by/ $4.0 /)$.

\begin{abstract}
The use of probiotic microorganisms in clinical practice has increased in recent years and a significant number of pregnant women are regular consumers of these products. However, probiotics might modulate the immune system, and whether or not this modulation is beneficial for perinatal outcomes is unclear. We performed a systematic review and meta-analysis to evaluate the reporting of perinatal outcomes in randomized controlled trials including women supplemented with probiotic microorganisms during pregnancy. We also analyzed the effects that the administration of probiotic microorganisms exerts on perinatal outcomes. In the review, 46 papers were included and 25 were meta-analyzed. Reporting of perinatal outcomes was highly inconsistent across the studies. Only birth weight, cesarean section, and weeks of gestation were reported in more than $50 \%$ of the studies. Random effects meta-analysis results showed that the administration of probiotic microorganisms during pregnancy did not have any a positive or negative impact on the perinatal outcomes evaluated. Subgroup analysis results at the strain level were not significantly different from main analysis results. The administration of probiotic microorganisms does not appear to influence perinatal outcomes. Nonetheless, future probiotic studies conducted in pregnant women should report probiotic strains and perinatal outcomes in order to shed light upon probiotics' effects on pregnancy outcomes.
\end{abstract}

Keywords: probiotics; safety; pregnancy; perinatal outcomes; strains; meta-analysis; PRISMA

\section{Introduction}

According to the World Health Organization (WHO), Food and Agriculture Organization (FAO), and the International Scientific Association for Probiotics and Prebiotics (ISAPP), probiotics are defined as "live microorganisms which when administered in adequate amounts confer a health benefit on the host" [1,2]. Similarly, synbiotic products are combinations of probiotic microorganisms and a beneficial substrate constituted by the prebiotic [3]. The use of probiotic microorganisms is widespread, and probiotic/synbiotic products are available in the global market labeled as food supplements or medical products depending on different regulations [4]. The market value of these products was estimated to account for USD 54 billion in 2020 and this trend suggests that it will grow substantially in the next years [5].

Probiotic microorganisms have been used for a plethora of clinical indications such as lactose malabsorption, diarrhea, bowel syndrome, and infection, among others [6]. In pregnant women, the administration of these microorganisms has been proposed to alleviate gastrointestinal symptoms, improve glycemic control, reduce oxidative stress, and lower the incidence of asthma, atopic sensitization, and allergic disease in offspring, among other outcomes [7-9]. However, clinical evidence remains far from conclusive [10]. 
Pregnancy is a dynamic immunological process in which pro- and anti-inflammatory status concur in order to facilitate the different stages of gestation [11]. Whereas a proinflammatory stage is necessary for successful embryo implantation and placentation during early pregnancy, an anti-inflammatory switch is required to allow fetal growth during mid-pregnancy. Finally, once fetal development is complete, another physiological pro-inflammatory status leads to labor and delivery [11]. Disruption of this immunological process is linked to adverse perinatal outcomes such as miscarriage, intrauterine growth restriction, and preterm birth [12].

Immune stimulation is among the different mechanisms of action attributed to probiotic microorganisms [13]. One study observed that pregnant women supplemented with a combination of Bifidobacterium longum, Lactobacillus delbrueckii, and Streptococcus thermophilus from 32 weeks of gestation to delivery had higher concentrations of proinflammatory cytokines, namely interleukine-5 (IL-5), interleukine-6 (IL-6), tumor necrosis factor- $\alpha$ (TNF- $\alpha$ ), and granulocyte-macrophage colony-stimulating factor (GM-CSF) [14]. On the other hand, a recent study monitored populations of immune cells after the administration of Lactobacillus reuteri to pregnant women during mid-gestation, observing that the number of activated regulatory $\mathrm{T}$ cells was lower in the group supplemented with Lactobacillus reuteri in comparison to the placebo [15]. Hence, the administration of probiotic microorganisms can potentially stimulate or suppress inflammatory status and immune response during gestation, but whether or not these changes are beneficial to pregnancy is unclear $[14,16]$.

Previous reviews have evaluated the effect that the administration of probiotic microorganisms during pregnancy exerts on maternal and perinatal outcomes, concluding that probiotic microorganisms do not increase or decrease the incidence of these outcomes $[10,17,18]$. These studies have only evaluated these microorganisms at the genus or species level. However, mounting evidence suggests that probiotic effects are strain-dependent, and strain-specificity is usually a poorly reported aspect of probiotics research [19]. Furthermore, studies explicitly designed to assess the safety of probiotic/synbiotic interventions are lacking [20].

In the light of these studies and given the rapid growth of literature regarding probiotic microorganisms, we aimed to evaluate the reporting of perinatal outcomes in randomized controlled trials (RCT) studying the administration of probiotic microorganisms during pregnancy and to explore the associations between the administration of probiotic microorganisms during pregnancy and perinatal outcomes considering the role that strain-specificity could play in the associations.

\section{Materials and Methods}

We conducted a systematic review and meta-analysis of randomized controlled trials studying the administration of probiotic microorganisms during pregnancy. The present systematic review was conducted according to the Preferred Reporting Items for Systematic Reviews and Meta-Analyses statement (PRISMA) [21]. The protocol for this study was registered in the PROSPERO database with the number "CRD42020216531." The PRISMA checklist is presented in Supplementary 5.

\subsection{Literature Search}

We carried out an automatized search in three databases, PubMed (MEDLINE), Scopus, and Cochrane Controlled Register of Trials (CENTRAL), during August-October 2020. The records dated from inception up to August 2020. The databases were searched by one reviewer. The language was restricted to English, and only full articles published in scientific journals were included in the research. A detailed search strategy based on patients, interventions, comparators, outcomes, and study design (PICOS) chosen for the present study is presented in Supplementary 1. 


\subsection{Inclusion and Exclusion Criteria}

We selected randomized controlled trials enrolling pregnant women in which at least one group of study was treated with probiotic or synbiotic preparations, with specified microorganisms and dosage, and at least one group of comparison received no treatment, routine care, or placebo, independently of the blinding process.

We excluded grey literature such as conference abstracts, workshops, and government reports, as well as other study designs. However, references from systematic reviews were searched for additional articles. Trials studying fermented food or generic products (i.e., probiotic food or probiotic supplement) without specifying the dosage and microorganisms provided were excluded. Studies comparing the use of probiotic preparations against antibiotics, as well as studies without follow-up to delivery, were also excluded. Secondary analyses of previous studies were not included in the review, but they were screened for additional information when not reported in the main analysis and were thus cited in tables when appropriate.

\subsection{Study Outcomes}

The prespecified main outcomes of the study consisted of the number of weeks of gestation, birth weight, preterm birth, cesarean section, low birth weight, macrosomia, small for gestational age (SGA), large for gestational age (LGA), miscarriage, and stillbirth. Secondary outcomes reported in the studies, namely cases of malformation, Apgar test score, umbilical cord $\mathrm{pH}$, anthropometric measures at birth, neonatal death, and admission to neonatal intensive care unit, were also discussed but were not considered for meta-analysis.

Preterm birth was defined as a baby born alive before 37 completed weeks of gestation [22]. SGA was defined as a live birth with a weight below the 10th percentile for the gestation age, while LGA was defined as a live birth with a weight above the 90th percentile [23]. According to ICD-10, low birth weight was considered as a live birth weighing less than $2500 \mathrm{~g}$ at delivery [24], while macrosomia was defined as a live birth weighing more than $4000 \mathrm{~g}$ at delivery in accordance with the American College of Obstetricians and Gynecologists [25]. When authors provided different definitions for these outcomes, they were specified in tables (Supplementary 3: Table S2).

\subsection{Data Extraction}

A template was created for data extraction, and two reviewers independently extracted the data from the included studies. The template consisted of the following items: Authors, year of acceptance for publication, country where the study was conducted, study design, sample size at randomization, sample size of groups analyzed, specific previous conditions (i.e., women with gestational diabetes mellitus), comparison group, main objective of the study, probiotic microorganisms, dosage and posology, vehicle of administration, duration of treatment, conflict of interest, and raw data or statistics on the reported perinatal outcomes (i.e., mean and standard deviation).

Studies using probiotic/synbiotic products provided by a company without stating the manufacturer's contribution to the paper were considered to present potential conflicts of interest. Disagreements between both reviewers were resolved by a third reviewer.

\subsection{Quality Assessment}

Two reviewers independently evaluated the quality of the included studies using the Cochrane risk-of-bias tool for randomized trials (RoB 2.0) [26]. Five domains were evaluated, including the randomization process, deviations from intended interventions, missing outcome data, measurement of the outcome, and selection of reported results. Follow-up losses $>20 \%$ were considered as a high risk of bias when assessing missing outcome data. Protocols of included studies were checked when assessing selective report bias. Regarding the overall risk of bias, we applied the following scheme based on the Cochrane Handbook recommendations [27]: One item rated as "high risk of bias" or three 
or more items rated as "some concerns" = high risk of bias; one or two items rated as "some concerns" = some concerns; all items rated as "low risk of bias" = low risk of bias. Any disagreement was discussed with a third reviewer.

\subsection{Data Synthesis and Statistical Analysis}

We conducted a random effects meta-analysis of the studies included in the review based on criteria selected to avoid potential bias when pooling results. Selected inclusion criteria for the meta-analysis were as follows: (1) Study population consisting of pregnant women without specified previous pathologies, and (2) studies reporting raw data or statistics on the specific perinatal outcome. We did not exclude women at high risk of atopic sensitization from the meta-analysis.

Groups of study assessing additional interventions other than dietary advice (i.e., probiotics + vitamin D) were excluded from analyses. If one study reported two or more groups treated with different probiotic microorganisms, they were combined. Data were pooled as relative risk and $95 \%$ confidence interval $(95 \% \mathrm{CI})$ or mean \pm standard deviation (SD), and the $\mathrm{I}^{2}$ statistic was used to assess heterogeneity across the studies. We used the Mantel-Haenszel statistical method in all of the analyses. Data reported as mean $\pm 95 \% \mathrm{CI}$ were transformed into mean $\pm \mathrm{SD}$. These approaches are recommended by the Cochrane handbook [27]. When data were reported as median and interquartile range or median and range, they were transformed into mean $\pm \mathrm{SD}$ using the method developed by Wan et al. [28]. Additionally, we provided subgroup analyses excluding these estimated statistics in order to assess how these approaches could influence pooled results (Supplementary 4).

Only outcomes reported in at least two trials that met the aforementioned inclusion criteria were pooled. When preterm birth cases were considered as an exclusion criterium in the papers, we added those preterm birth cases to the groups analyzed. There was only one paper studying synbiotic products that met the inclusion criteria. Therefore, only probiotic studies were finally meta-analyzed.

Subgroup analyses were conducted based on reported strains of microorganisms. Accordingly, we pooled data on outcomes reported in at least two studies using the same microorganism strain or the same combination of strains.

Finally, we created funnel plots for each main analysis including at least 10 studies. Publication bias was assessed by visual inspection of the funnel plots.

All analyses were conducted using Review Manager (RevMan), version 5.4, The Cochrane Collaboration, 2020.

\section{Results}

\subsection{Selection Process}

A total of 26,579 records were screened after removing duplicates, leaving 115 articles available for eligibility, including 10 references from other previous reviews. Of the eligible articles, 87 met the inclusion criteria and were further evaluated to exclude any secondary analysis of a previous research. Finally, 46 RCTs were included in the systematic review and 25 RCTs were included in the meta-analysis. Secondary analyses of previous studies were screened for additional data if they were not reported in the original research, and thus were cited in tables when applicable. The article selection process is presented in Figure 1 according to the PRISMA flow diagram of trials. 


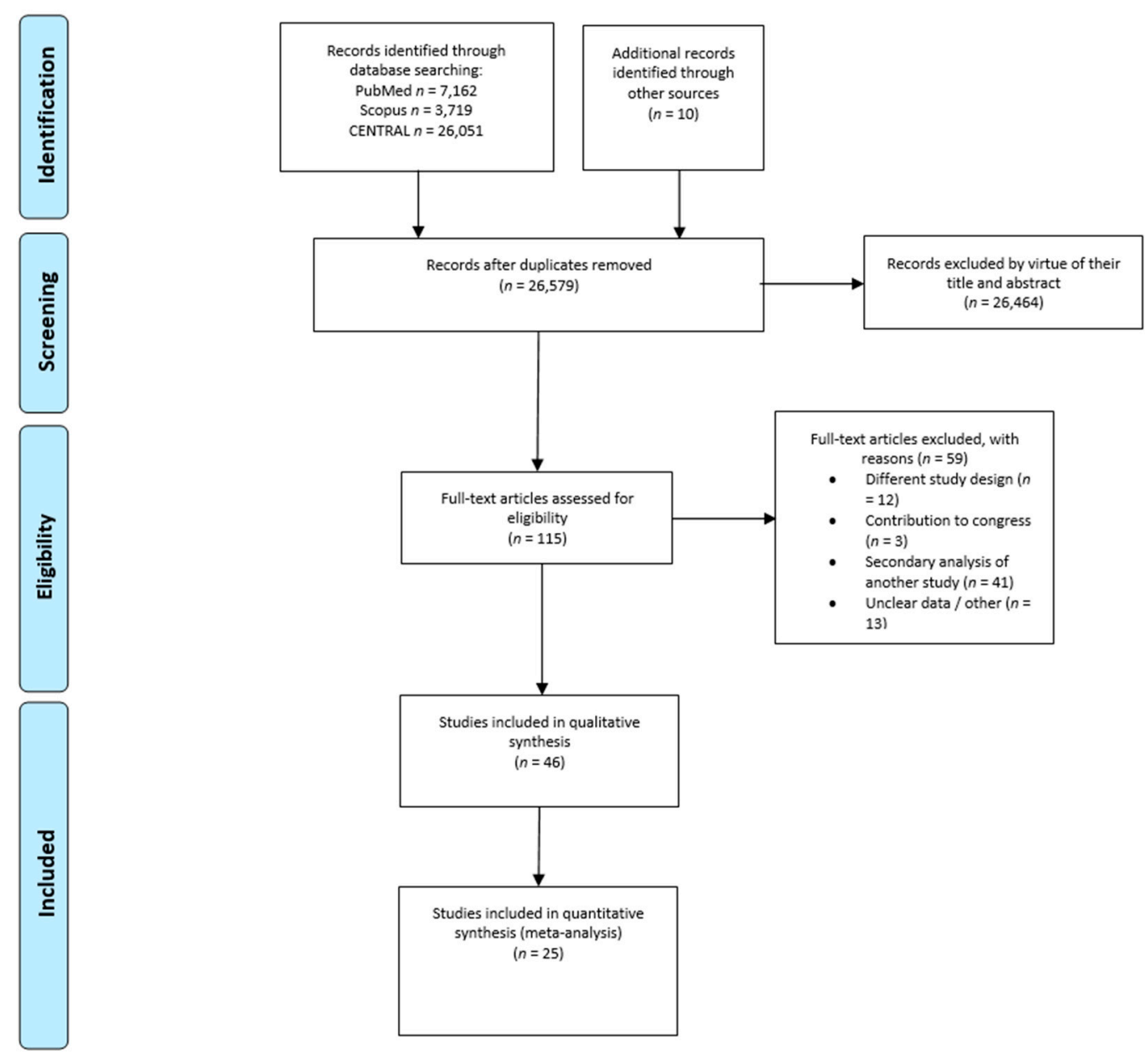

Figure 1. Preferred Reporting Items for Systematic Reviews and Meta-Analysis (PRISMA) flow diagram of trials.

\subsection{Characteristics of the Studies}

Characteristics of studies included in the systematic review are presented in Table 1. 
Table 1. Characteristics of the included studies.

\begin{tabular}{|c|c|c|c|c|c|c|c|c|}
\hline $\begin{array}{c}\text { Author/Country/Year of } \\
\text { Acceptance for } \\
\text { Publication/Trial Registration }\end{array}$ & $\begin{array}{l}\text { Sample Size at } \\
\text { Randomization }\end{array}$ & $\begin{array}{l}\text { Groups Analyzed at } \\
\text { Delivery }\end{array}$ & $\begin{array}{l}\text { Microorganism (Strain)/Dosage (Posology) } \\
\text { + Additional Interventions }\end{array}$ & $\begin{array}{l}\text { Administration } \\
\text { Vehicle }\end{array}$ & Treatment Duration & $\begin{array}{l}\text { Main Clinical } \\
\text { Outcome }\end{array}$ & $\begin{array}{l}\text { Risk of Bias } \\
\text { Assessment }\end{array}$ & $\begin{array}{l}\text { Conflict of } \\
\text { Interest }\end{array}$ \\
\hline $\begin{array}{l}\text { Abrahamsson } \\
\text { et al. } / \text { Sweden/2007/Not } \\
\text { reported [29] }\end{array}$ & 232 & $\begin{array}{l}\text { Probiotic }=95 \\
\text { Placebo }=93\end{array}$ & $\begin{array}{l}\text { Lactobacillus reuteri (ATCC 55730)/1 } 1 \times 10^{8} \mathrm{CFU} \\
\text { (daily) }\end{array}$ & Oil & $\begin{array}{c}\text { From } 36 \text { WoG to } \\
\text { delivery }\end{array}$ & $\begin{array}{l}\text { Eczema and } \\
\text { sensitization in } \\
\text { offspring }\end{array}$ & Low & $\begin{array}{l}\text { Existing conflict of } \\
\text { interest }\end{array}$ \\
\hline $\begin{array}{c}\text { Ahmadi et al./ } \\
\text { Iran/2016/IRCT2016050 } \\
\text { 85623N77 [30] }\end{array}$ & 70 & $\begin{array}{l}\text { Synbiotic }=35 \\
\text { Placebo }=35\end{array}$ & $\begin{array}{c}\text { Lactobacillus acidophilus } / 2 \times 10^{8} \mathrm{CFU} \\
\text { Lactobacillus casei } / 2 \times 10^{8} \mathrm{CFU} \\
\text { Bifidobacterium bifidum } / 2 \times 10^{8} \mathrm{CFU} \\
+ \text { Inulin/0.8 } \\
\text { (daily) }\end{array}$ & Oral capsule & $\begin{array}{l}\text { Six weeks from 24-28 } \\
\text { WoG }\end{array}$ & $\begin{array}{l}\text { Insulin metabolism } \\
\text { and lipid profile }\end{array}$ & Low & $\begin{array}{l}\text { Potential conflict } \\
\text { of interest }\end{array}$ \\
\hline $\begin{array}{c}\text { Allen et al./United King- } \\
\text { dom/2009/ISRCTN26287422 } \\
\text { [32] }\end{array}$ & 454 & $\begin{array}{l}\text { Probiotic }=220 \\
\text { Placebo }=234\end{array}$ & $\begin{array}{c}\text { Lactobacillus salivarius (CUL61, NCIMB 30211)/ } \\
6.25 \times 10^{9} \mathrm{CFU} \\
\text { Lactobacillus paracasei (CUL08, NCIMB 30154) } \\
/ 1.25 \times 10^{9} \mathrm{CFU} \\
\text { Bifidobacterium animalis (CUL34, NCIMB } \\
30172) / 1.25 \times 10^{9} \mathrm{CFU} \\
\text { Bifidobacterium bifidum (CUL20, NCIMB 30153)/ } \\
1.25 \times 10^{9} \mathrm{CFU} \text { (daily) }\end{array}$ & Oral capsule & $\begin{array}{l}\text { From last month of } \\
\text { gestation to } \\
\text { postpartum }\end{array}$ & $\begin{array}{l}\text { Prevalence of } \\
\text { adverse events }\end{array}$ & High & $\begin{array}{l}\text { Existing conflict of } \\
\text { interest }\end{array}$ \\
\hline $\begin{array}{l}\text { Asemi et al./Iran } / 2011 / \text { Not } \\
\text { reported [33] }\end{array}$ & 82 & $\begin{array}{l}\text { Probiotic }=37 \\
\text { Control }=33\end{array}$ & $\begin{array}{l}\text { Lactobacillus acidophilus (LA-5) } / 1 \times 10^{7} \mathrm{CFU} \\
\text { Bifidobacterium animalis (Bb-12)/1 } \times 10^{7} \mathrm{CFU} \\
\text { (200 g daily) }\end{array}$ & Yoghurt & $\begin{array}{l}\text { Nine weeks from the } \\
\text { third trimester of } \\
\text { gestation }\end{array}$ & $\begin{array}{l}\text { Insulin resistance } \\
\text { and insulin levels }\end{array}$ & High & $\begin{array}{l}\text { Potential conflict } \\
\text { of interest }\end{array}$ \\
\hline $\begin{array}{c}\text { Asgharian et al./ } \\
\text { Iran/2019//RCT201604013 } \\
\text { 706N31 [34] } \\
\end{array}$ & 130 & $\begin{array}{l}\text { Probiotic }=64 \\
\text { Control }=64\end{array}$ & $\begin{array}{l}\text { Lactobacillus acidophilus (LA-5)/5 } / 510^{8} \mathrm{CFU} / \mathrm{g} \\
\text { Bifidobacterium animalis (Bb-12)/5 } \times 10^{8} \mathrm{CFU} / \mathrm{g} \\
\text { (100 g daily) }\end{array}$ & Yoghurt & $\begin{array}{l}\text { From } 24 \text { WoG to } \\
\text { delivery }\end{array}$ & Glucose levels & Low & $\begin{array}{l}\text { Potential conflict } \\
\text { of interest }\end{array}$ \\
\hline $\begin{array}{c}\text { Bababi et al./ } \\
\text { Iran/2008/IRCT2017101003 } \\
\text { 6697N1 [35] }\end{array}$ & $\begin{array}{l}50 \text { pregnant } \\
\text { women with } \\
\text { GDM }\end{array}$ & $\begin{array}{l}\text { Probiotic }=24 \\
\text { Control }=24\end{array}$ & $\begin{array}{l}\text { Lactobacillus acidophilus/2 } \times 10^{9} \mathrm{CFU} / \mathrm{g} \\
\text { Lactobacillus casei/2 } 2 \times 10^{9} \mathrm{CFU} / \mathrm{g} \\
\text { Bifidobacterium bidifum } / 2 \times 10^{9} \mathrm{CFU} / \mathrm{g} \\
\text { Lactobacillus fermentum } / 2 \times 10^{9} \mathrm{CFU} / \mathrm{g} \\
\text { (posology not clearly defined) }\end{array}$ & Oral capsule & $\begin{array}{l}\text { Six weeks from 24-28 } \\
\text { WoG }\end{array}$ & $\begin{array}{l}\text { Genetic and } \\
\text { metabolic profile }\end{array}$ & Low & $\begin{array}{l}\text { Potential conflict } \\
\text { of interest }\end{array}$ \\
\hline $\begin{array}{l}\text { Badehnoosh et al./ } \\
\text { Iran/2017/IRCT201611115 } \\
\text { 623N91 [36] }\end{array}$ & $\begin{array}{l}60 \text { pregnant } \\
\text { women with } \\
\text { GDM }\end{array}$ & $\begin{array}{c}\text { Probiotic }=30 \\
\text { Placebo }=30\end{array}$ & $\begin{array}{c}\text { Lactobacillus acidophilus } / 2 \times 10^{9} \mathrm{CFU} / \mathrm{g} \\
\text { Lactobacillus casei } / 2 \times 10^{9} \mathrm{CFU} / \mathrm{g} \\
\text { Bifidobacterium bidifum } / 2 \times 10^{9} \mathrm{CFU} / \mathrm{g} \\
\text { (Posology not clearly defined) }\end{array}$ & Oral capsule & $\begin{array}{c}\text { Six weeks from } 24-28 \\
\text { WoG }\end{array}$ & $\begin{array}{l}\text { Oxidative stress and } \\
\text { inflammation } \\
\text { biomarkers }\end{array}$ & Low & $\begin{array}{l}\text { Potential conflict } \\
\text { of interest }\end{array}$ \\
\hline $\begin{array}{c}\text { Boyle } \\
\text { et al./Australia/2008/Cochrane } \\
\text { Skin Group Trial No. } 36 \text { [37] }\end{array}$ & 250 & $\begin{array}{l}\text { Probiotic }=125 \\
\text { Placebo }=125\end{array}$ & $\begin{array}{c}\text { Lactobacillus rhamnosus (GG) } / 1.8 \times 10^{10} \mathrm{CFU} \\
\text { (daily) }\end{array}$ & Oral capsule & $\begin{array}{l}\text { From } 36 \text { WoG to } \\
\text { delivery }\end{array}$ & $\begin{array}{l}\text { Risk of eczema } \\
\text { during infancy }\end{array}$ & Low & $\begin{array}{l}\text { Potential conflict } \\
\text { of interest }\end{array}$ \\
\hline
\end{tabular}


Table 1. Cont.

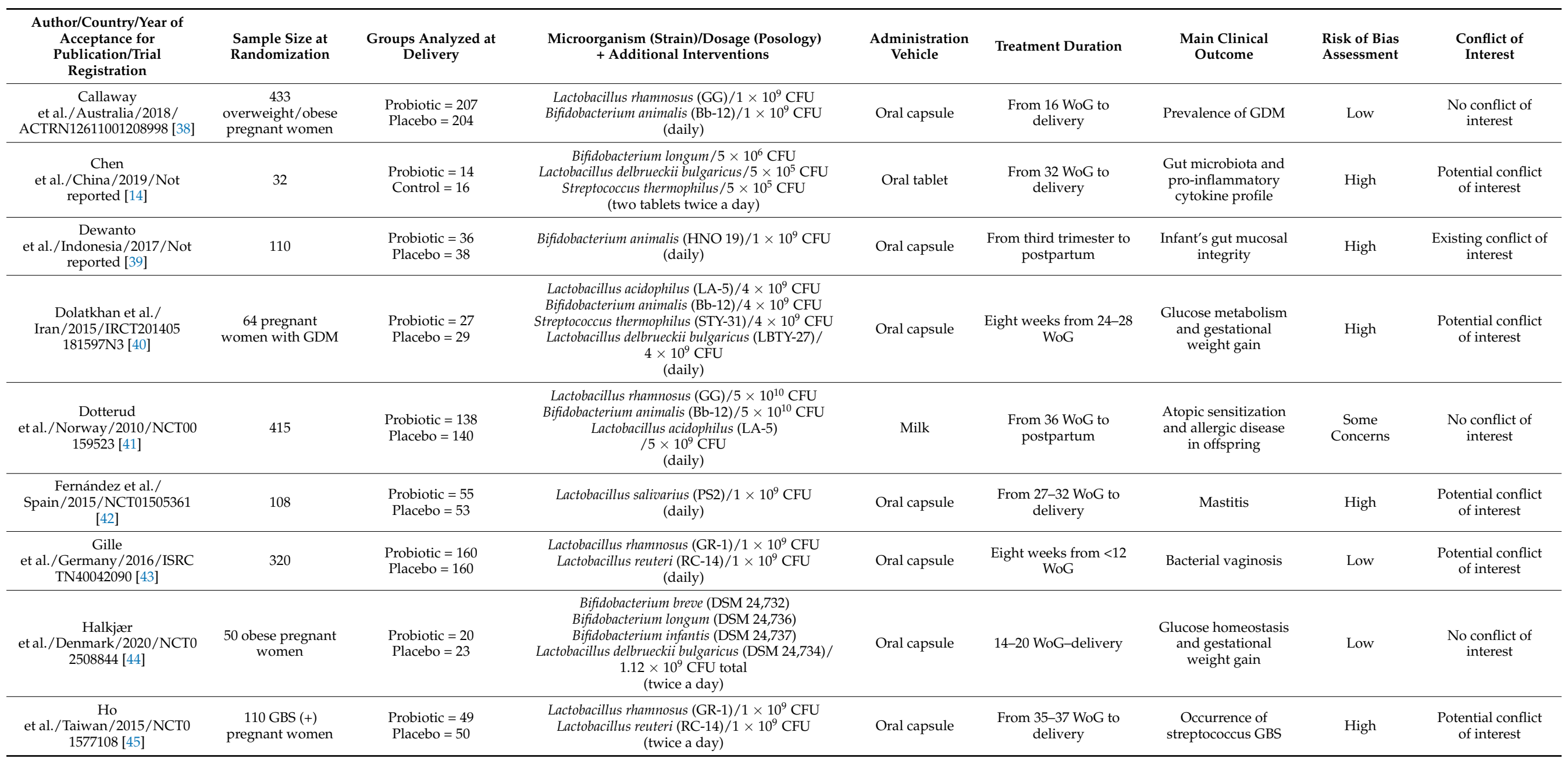


Table 1. Cont

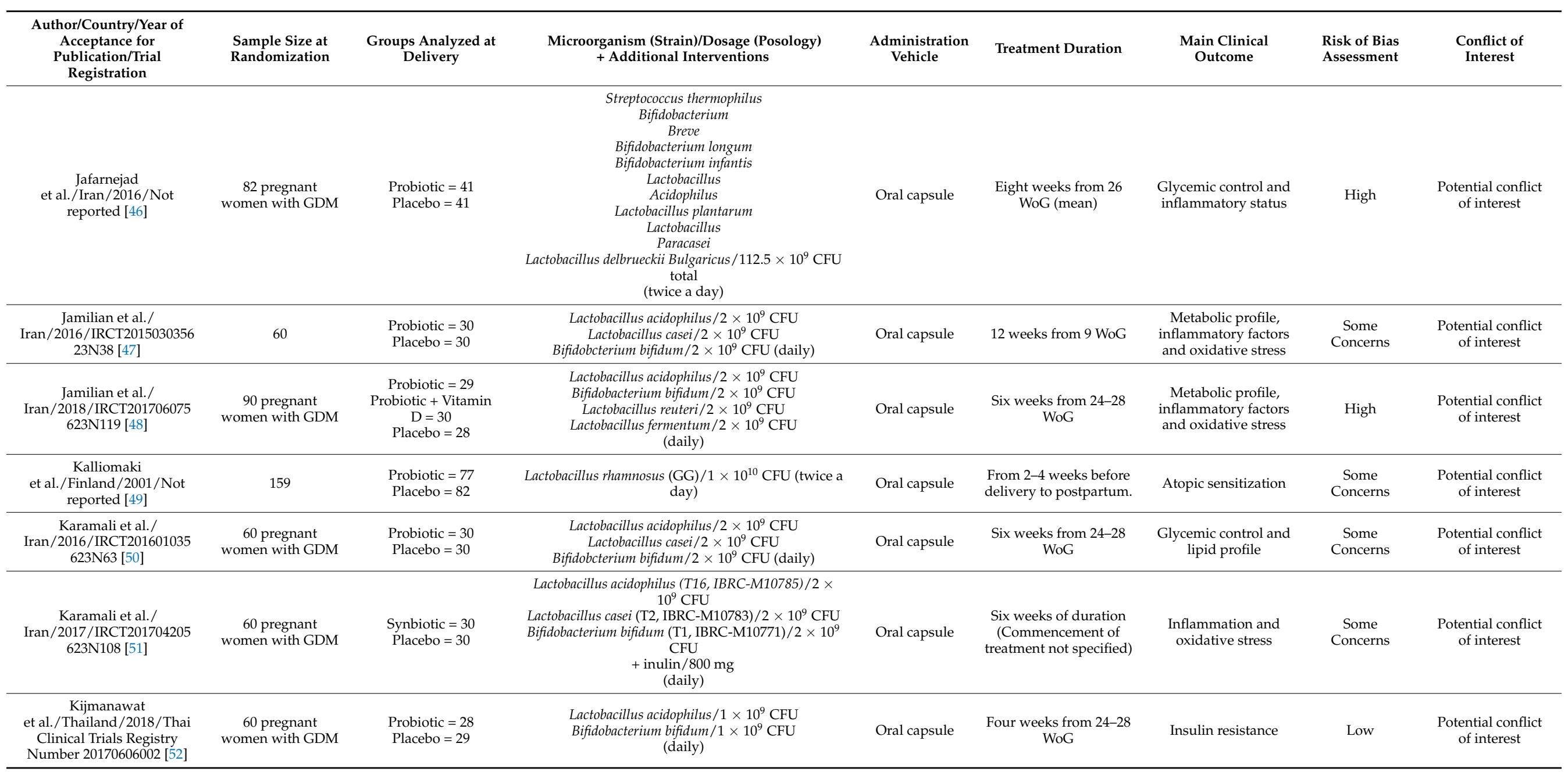


Table 1. Cont.

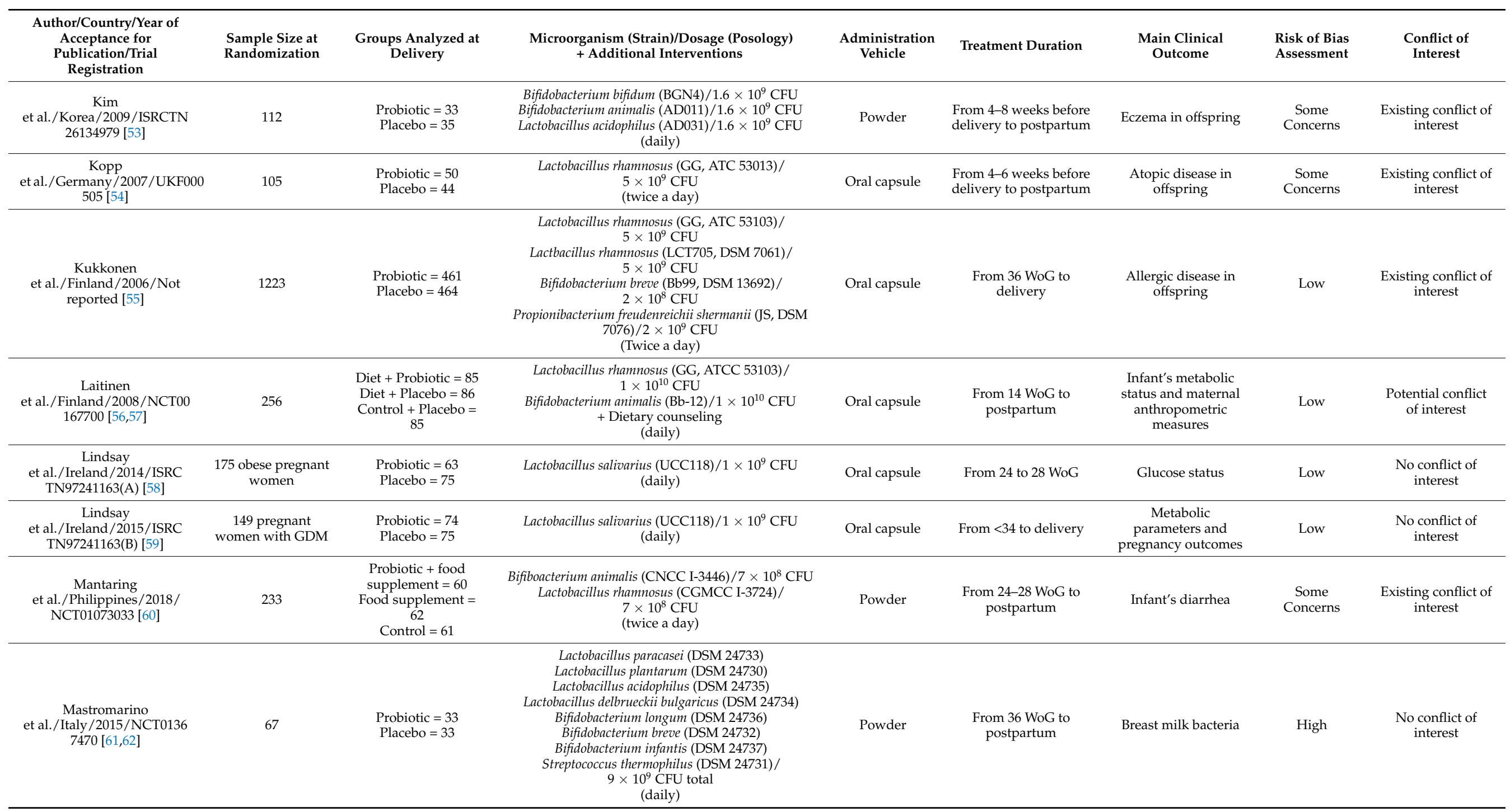


Table 1. Cont.

\begin{tabular}{|c|c|c|c|c|c|c|c|c|}
\hline $\begin{array}{l}\text { Author/Country/Year of } \\
\text { Acceptance for } \\
\text { Publication/Trial } \\
\text { Registration }\end{array}$ & $\begin{array}{l}\text { Sample Size at } \\
\text { Randomization }\end{array}$ & $\begin{array}{l}\text { Groups Analyzed at } \\
\text { Delivery }\end{array}$ & $\begin{array}{l}\text { Microorganism (Strain)/Dosage (Posology) } \\
\text { + Additional Interventions }\end{array}$ & $\begin{array}{l}\text { Administration } \\
\text { Vehicle }\end{array}$ & Treatment Duration & $\begin{array}{l}\text { Main Clinical } \\
\text { Outcome }\end{array}$ & $\begin{array}{l}\text { Risk of Bias } \\
\text { Assessment }\end{array}$ & $\begin{array}{l}\text { Conflict of } \\
\text { Interest }\end{array}$ \\
\hline $\begin{array}{c}\text { McMillan et al./ } \\
\text { Rwanda/2018/NCT02150655 } \\
\text { [63] }\end{array}$ & 38 & $\begin{array}{l}\text { Probiotic }=8 \\
\text { Placebo }=5\end{array}$ & $\begin{array}{c}\text { Lactobacillus rhamnosus (GR-1)/1 } \times 10^{9} \mathrm{CFU} \\
\text { Lactobacillus reuteri (RC-14)/1 } \times 10^{9} \mathrm{CFU} \\
\text { (daily) }\end{array}$ & Oral capsule & $\begin{array}{l}\text { One month from 4-32 } \\
\text { WoG }\end{array}$ & Vaginal microbiota & High & $\begin{array}{l}\text { Potential conflict } \\
\text { of interest }\end{array}$ \\
\hline $\begin{array}{c}\text { Nabhani et al./ } \\
\text { Iran/2018/IRCT201511183 } \\
\text { 140N16 [64] }\end{array}$ & $\begin{array}{l}95 \text { pregnant } \\
\text { women with GDM }\end{array}$ & $\begin{array}{l}\text { Synbiotic }=45 \\
\text { Placebo }=45\end{array}$ & $\begin{array}{c}\text { Lactobacillus acidophilus } / 5 \times 10^{10} \mathrm{CFU} / \mathrm{g} \\
\text { Lactobacillus plantarum } / 1.5 \times 10^{10} \mathrm{CFU} / \mathrm{g} \\
\text { Lactobacillus fermentum } / 7 \times 10^{9} \mathrm{CFU} / \mathrm{g} \\
\text { Lactobacillus gasseri } / 2 \times 10^{10} \mathrm{CFU} / \mathrm{g} \\
+\mathrm{FOS} / 38.5 \mathrm{mg} \\
\text { (500 } \mathrm{mg} \text { daily) }\end{array}$ & Oral capsule & $\begin{array}{l}\text { Six weeks from } 24-28 \\
\text { WoG }\end{array}$ & $\begin{array}{l}\text { Insulin resistance, } \\
\text { lipid profile and } \\
\text { antioxidative status }\end{array}$ & $\begin{array}{l}\text { Some } \\
\text { Concerns }\end{array}$ & $\begin{array}{l}\text { Potential conflict } \\
\text { of interest }\end{array}$ \\
\hline $\begin{array}{l}\text { Niers } \\
\text { et al./Netherlands/2009/NCT } \\
\text { 00200954 [65] }\end{array}$ & 156 & $\begin{array}{l}\text { Probiotic }=50 \\
\text { Placebo }=52\end{array}$ & $\begin{array}{c}\text { Bifidobacterium bifidum }(\mathrm{W} 23) / 1 \times 10^{9} \mathrm{CFU} \\
\text { Bifidobacterium animalis (W52)/1 } \times 10^{9} \mathrm{CFU} \\
\text { Lactococcus lactis (W58)/1 } \times 10^{9} \mathrm{CFU} \\
\text { (daily) }\end{array}$ & Oral capsule & $\begin{array}{l}\text { From the last six weeks } \\
\text { of pregnancy to } \\
\text { postpartum }\end{array}$ & $\begin{array}{l}\text { Eczema in offspring, } \\
\text { microbial } \\
\text { colonization and } \\
\text { immune response }\end{array}$ & $\begin{array}{l}\text { Some } \\
\text { Concerns }\end{array}$ & $\begin{array}{l}\text { Existing conflict of } \\
\text { interest }\end{array}$ \\
\hline $\begin{array}{c}\text { Okense-Gafa et al./New } \\
\text { Zealand/2018/ACTRN12615 } \\
\text { 000400561 [66] }\end{array}$ & $\begin{array}{l}230 \text { obese pregnant } \\
\text { women }\end{array}$ & $\begin{array}{l}\text { Probiotic }=115 \\
\text { Placebo }=115\end{array}$ & $\begin{array}{c}\text { Lactobacillus rhamnosus (GG) } / 7 \times 10^{9} \mathrm{CFU} \\
\text { Bifidobacterium animalis (Bb-12)/7 } \times 10^{9} \mathrm{CFU} \\
\text { (daily) }\end{array}$ & Oral capsule & $\begin{array}{l}\text { From } 12-17 \text { WoG to } \\
\text { delivery }\end{array}$ & $\begin{array}{l}\text { Gestational weight } \\
\text { gain and pregnancy } \\
\text { outcomes }\end{array}$ & Low & $\begin{array}{l}\text { No conflict of } \\
\text { interest }\end{array}$ \\
\hline $\begin{array}{c}\text { Olsen } \\
\text { et al./ Australia/2017/Not } \\
\text { reported [67] }\end{array}$ & 34 & $\begin{array}{l}\text { Probiotic }=7 \\
\text { Control }=13\end{array}$ & $\begin{array}{l}\text { Lactobacillus rhamnosus (GR-1) } \\
\text { Lactobacillus reuteri (RC-14)/1 } \times 10^{8} \text { CFU total } \\
\text { (daily) }\end{array}$ & Not specified & $\begin{array}{l}\text { Three weeks from } 36 \\
\text { WoG }\end{array}$ & $\begin{array}{l}\text { Occurrence of } \\
\text { streptococcus GBS }\end{array}$ & High & $\begin{array}{l}\text { Potential conflict } \\
\text { of interest }\end{array}$ \\
\hline $\begin{array}{l}\text { Pellonperä } \\
\text { et./Finland/2019/NCT01922791 } \\
\text { [69] }\end{array}$ & 439 & $\begin{array}{c}\text { Probiotic }+ \text { Placebo }= \\
110 \\
\text { Fish oil }+ \text { Probiotic }= \\
109 \\
\text { Fish oil }+ \text { Placebo }= \\
109 \\
\text { Placebo }+ \text { Placebo }= \\
110\end{array}$ & $\begin{array}{c}\text { Lactobacillus rhamnosus (HNO01, ATCC SD5675)/ } \\
1 \times 10^{10} \mathrm{CFU} \\
\text { Bifidobacterium animalis }(420, \mathrm{DSM} 22089) / \\
1 \times 10^{10} \mathrm{CFU} \\
(\text { daily) }\end{array}$ & Oral capsule & $\begin{array}{l}\text { From }<18 \text { WoG to } \\
\text { postpartum }\end{array}$ & $\begin{array}{l}\text { Risk of GDM and } \\
\text { glucose metabolism }\end{array}$ & Low & $\begin{array}{l}\text { No conflict of } \\
\text { interest }\end{array}$ \\
\hline $\begin{array}{c}\text { Sahhaf et al./ } \\
\text { Iran/2019/IRCT201212240118 } \\
\text { 62N2 [70] } \\
\end{array}$ & 84 & $\begin{array}{l}\text { Probiotic }=42 \\
\text { Placebo }=42\end{array}$ & $\begin{array}{l}\text { Lactobacillus acidophilus } \\
\text { Bifidobacterium animalis } \\
/ 1 \times 10^{6} \mathrm{CFU} \text { total ( } 300 \mathrm{mg} \text { daily) }\end{array}$ & Yoghurt & $\begin{array}{l}\text { Eight weeks from } 24-28 \\
\text { WoG }\end{array}$ & Glycemic parameters & $\begin{array}{l}\text { Some } \\
\text { Concerns }\end{array}$ & $\begin{array}{l}\text { Potential conflict } \\
\text { of interest }\end{array}$ \\
\hline $\begin{array}{c}\text { Sharpe } \\
\text { et al./Canada/2019/NCT025 } \\
28981[71] \\
\end{array}$ & 139 & $\begin{array}{l}\text { Probiotic }=57 \\
\text { Placebo }=56\end{array}$ & $\begin{array}{l}\text { Lactobacillus rhamnosus (GR-1)/2.5 } \times 10^{9} \mathrm{CFU} \\
\text { Lactobacillus reuteri (RC-14)/2.5 } \times 10^{9} \mathrm{CFU} \text { (twice } \\
\text { a day) }\end{array}$ & Oral capsule & $\begin{array}{l}\text { Twelve weeks from } \\
23-25 \text { WoG }\end{array}$ & $\begin{array}{l}\text { Occurrence of } \\
\text { streptococcus GBS }\end{array}$ & $\begin{array}{l}\text { Some } \\
\text { Concerns }\end{array}$ & $\begin{array}{l}\text { Potential conflict } \\
\text { of interest }\end{array}$ \\
\hline $\begin{array}{c}\text { Taghizadeh et al./ } \\
\text { Iran/2013/IRCT20121210562 } \\
\text { 3N3 [72] }\end{array}$ & 56 & $\begin{array}{l}\text { Synbiotic }=26 \\
\text { Placebo }=26\end{array}$ & $\begin{array}{l}\text { Lactobacillus sporogenes } / 9 \times 10^{7} \mathrm{CFU} \\
+ \text { Inulin } / 0.36 \mathrm{~g} \\
\text { (twice a day) }\end{array}$ & Not specified & $\begin{array}{l}\text { Nine weeks from the } \\
\text { third trimester of } \\
\text { gestation }\end{array}$ & $\begin{array}{l}\text { Glycemic status and } \\
\text { C-reactive protein } \\
\text { sensitivity }\end{array}$ & $\begin{array}{c}\text { Some } \\
\text { Concerns }\end{array}$ & $\begin{array}{l}\text { Potential conflict } \\
\text { of interest }\end{array}$ \\
\hline
\end{tabular}


Table 1. Cont.

\begin{tabular}{|c|c|c|c|c|c|c|c|c|}
\hline $\begin{array}{l}\text { Author/Country/Year of } \\
\text { Acceptance for } \\
\text { Publication/Trial } \\
\text { Registration }\end{array}$ & $\begin{array}{l}\text { Sample Size at } \\
\text { Randomization }\end{array}$ & $\begin{array}{l}\text { Groups Analyzed at } \\
\text { Delivery }\end{array}$ & $\begin{array}{l}\text { Microorganism (Strain)/Dosage (Posology) } \\
\text { + Additional Interventions }\end{array}$ & $\begin{array}{l}\text { Administration } \\
\text { Vehicle }\end{array}$ & Treatment Duration & $\begin{array}{l}\text { Main Clinical } \\
\text { Outcome }\end{array}$ & $\begin{array}{l}\text { Risk of Bias } \\
\text { Assessment }\end{array}$ & $\begin{array}{l}\text { Conflict of } \\
\text { Interest }\end{array}$ \\
\hline $\begin{array}{l}\text { Wickens et al./New } \\
\text { Zealand/2008/ACTRN } \\
12607000518460 \text { [73] }\end{array}$ & 512 & $\begin{array}{c}\text { Probiotic } 1=157 \\
\text { Probiotic } 2=158 \\
\text { Placebo }=159\end{array}$ & $\begin{array}{c}\text { Probiotic } 1=\text { Lactobacillus rhamnosus (HN001)/ } \\
6 \times 10^{9} \mathrm{CFU} \\
\text { Probiotic } 2 \\
=\text { Bifidobacterium animalis animalis (HN019)/ } \\
9 \times 10^{9} \mathrm{CFU} \\
\text { (daily) }\end{array}$ & Oral capsule & $\begin{array}{l}\text { From } 35 \text { WoG to } \\
\text { postpartum }\end{array}$ & $\begin{array}{l}\text { Eczema and atopic } \\
\text { sensitization in } \\
\text { offspring }\end{array}$ & Low & $\begin{array}{l}\text { Existing conflict of } \\
\text { interest }\end{array}$ \\
\hline $\begin{array}{c}\text { Wickens et al./New } \\
\text { Zealand/2017/ACTRN12612 } \\
\text { 000196842 [74] }\end{array}$ & 423 & $\begin{array}{l}\text { Probiotic }=206 \\
\text { Placebo }=202\end{array}$ & $\begin{array}{c}\text { Lactobacillus } \\
\text { rhamnosus }\left(\mathrm{HNO01)} / 6 \times 10^{9} \mathrm{CFU}\right. \\
\text { (daily) }\end{array}$ & Oral capsule & $\begin{array}{l}\text { From } 14-16 \text { WoG to } \\
\text { postpartum }\end{array}$ & $\begin{array}{l}\text { Occurrence of } \\
\text { streptococcus GBS }\end{array}$ & $\begin{array}{l}\text { Some } \\
\text { Concerns }\end{array}$ & $\begin{array}{l}\text { No conflict of } \\
\text { interest }\end{array}$ \\
\hline $\begin{array}{c}\text { Yang } \\
\text { et al./Canada/2020/NCT01 } \\
697683 \text { [75] }\end{array}$ & 86 & $\begin{array}{l}\text { Probiotic }=41 \\
\text { Placebo }=43\end{array}$ & $\begin{array}{l}\text { Lactobacillus rhamnosus (GR-1)/2.5 } \times 10^{9} \mathrm{CFU} \\
\text { Lactobacillus reuteri (RC-14)/2.5 } \times 10^{9} \mathrm{CFU} \text { (daily) }\end{array}$ & Oral capsule & $\begin{array}{l}\text { Twelve weeks from } \\
12-16 \text { WoG }\end{array}$ & $\begin{array}{l}\text { Vaginal microbiota, } \\
\text { chemokines and } \\
\text { cytokines profile }\end{array}$ & High & $\begin{array}{l}\text { Potential conflict } \\
\text { of interest }\end{array}$ \\
\hline
\end{tabular}

CFU: Colony-Forming Unit; GBS: Group B Streptococcus; GDM: Gestational Diabetes Mellitus; FOS: Fructooligosaccharide. WoG: Week of Gestation. 
In the present review, 46 randomized controlled trials were included. A total of 8519 pregnant women participated in these studies. Of the analyzed studies, 42 trials evaluated the administration of probiotic preparations to pregnant women whereas 4 studies evaluated the administration of synbiotic products. The included papers dated from 2001 to 2020. Of the studies, 15 were conducted in Iran [30,31,33-36,40,46-48,50, 51,64,70,72]; 4 trials were conducted in Finland [49,55,56,69]; 3 in New Zealand [66,73, 74]; 3 in Australia [37,38,67]; 2 each in Canada [71,75], Germany [43,54], Taiwan [45, 68], and Ireland [58,59], and the remaining 13 RCTs were conducted in other different countries. Most of the studies recruited healthy pregnant women. However, 10 trials included only pregnant women with gestational diabetes mellitus $[35,36,40,46,48,50-52,59$, 64], 4 studies included exclusively obese/overweight pregnant women $[38,44,58,66]$, and 1 study included only pregnant women carrying group B streptococcus (GBS) [45].

Regarding the main objectives of the studies, the most common were improving insulin/glucose metabolism (13 studies) [30,33,34,40,44,46,50,52,58,64,69,70,72]; preventing eczema, allergic disease, or atopic sensitization (10 studies) [29,37,41,49,53-55,65,68,73]; improving oxidative stress status and inflammatory profile (8 studies) $[14,36,46-48,51,64]$; modifying the mother's or infant's microbiota (5 studies) [14,61,63,65,75]; and preventing GBS occurrence (4 studies) $[45,67,71,74]$. Other outcomes included bacterial vaginosis, mastitis, pregnancy outcomes, safety assessment, maternal anthropometric measures, infant colic, genetic profile, and infant diarrhea or gut integrity.

In respect to the microorganisms administered, none of the included studies evaluated the administration of probiotic yeasts (i.e., Saccharomyces bourlardii) to pregnant women. In the studies, 21 of the authors administered combinations of Bifidobacterium and Lactobacillus species [30,32-36,38,41,44,47,48,50-53,56,60,66,67,69,73]; 18 authors studied the administration of Lactobacillus species only $[29,31,37,42,43,45,49,54,58,59,63,64,67$, 68,71,72,74,75]; 4 authors used combinations of Lactobacillus, Bifidobacterium, and Streptococcus species [14,40,46,61]; 1 author evaluated Bifidobacterium species only [39]; and the 2 remaining authors evaluated other combinations with different bacterial genera (Propionibacterium and Lactococcus) $[55,65]$. Strains of microorganisms administered were reported in 32 of the 46 included studies (70\%). The reporting of strains was heterogeneous across the studies, with authors reporting culture collection numbers (i.e., Lactobacillus rhamnosus ATCC 53103), commercial designations (i.e., Bifidobacterium animalis HN019), or references to the name of the person who originally isolated the strain (i.e., Lactobacillus rhamnosus GG). The most frequently used strains, alone or in combination with other strains, were L. rhamnosus GG (nine studies) [37,38,41,49,54-56,60,68], B.animalis BB-12 (seven studies) $[33,34,38,40,41,56,66]$, L. rhamnosus GR-1 (six studies) $[43,45,63,67,71,75]$, L. reuteri RC-14 [43,45,63,67,71,75], L. acidophilus LA-5 (four studies) [33,34,40,41], and L. rhamnosus HN001 (three studies) [69,73,74].

Probiotic/synbiotic administration was only compared to placebo in 37 trials (80\%) [2932,36-47,49-55,58,59,63-66,68,70-75], while 5 studies (11\%) used a group with no treatment or routine care as a comparison [14,33-35,67], and the remaining 4 papers (9\%) analyzed other additional comparison groups (i.e., probiotic + vitamin D) $[48,56,60,69]$. Microorganisms were administered orally in all of the included studies. Administration vehicles consisted of capsules in most of the cases (35 studies, $76 \%$ ), with 3 studies using probiotic yoghurt (6.5\%) [33,34,70]; 3 studies using probiotic powder [53,60,61]; and 1 study each using probiotic oil (2.2\%) [29], tablets [14], and milk [41]. The two remaining studies (4.3\%) did not specify the vehicle used to administer the microorganisms [67,72].

The dosages of individual microorganisms were diverse, ranging from $5 \times 10^{5} \mathrm{CFU}$ [14] to $5 \times 10^{10} \mathrm{CFU}$ [64]. However, in some cases, the posology was unclear [34,35]. In other cases, the dosages were defined as CFU/g, but the authors did not declare the mass of the product administered.

Mean treatment duration was approximately 9 weeks, ranging from 3 weeks of duration [67] to 26 weeks [56]. Women were treated with probiotic/synbiotic preparations during the third trimester of gestation in most of the trials (34 studies, 74\%) [2-37,39-42,45, 
$46,48,49,52-55,58-61,64,65,67,68,70-73]$, through second and third trimesters in 7 studies $(15 \%)[38,44,50,56,66,74,75]$, through first and second trimesters in 3 studies $[43,47,69]$, and independently of the trimester of gestation in 1 study [63]. One author did not specify the period of treatment [51].

Quality assessment results showed that 18 studies (39\%) had a low risk of bias (high methodological quality) [29-31,34-38,43,44,52,55,56,58,59,66,69,73], 15 studies (33\%) had an unclear risk of bias due to concerns in one or two of the items assessed [41,47,49$54,60,64,65,68,70,71,73,74]$, and the remaining 13 studies $(28 \%)$ had a high risk of bias (low methodological quality) $[14,32,33,39,40,42,45,46,48,61,63,67,75]$. Detailed results of the risk of bias assessment are presented in Supplementary 2: Table S1. Regarding conflicts of interest, 9 studies $(20 \%)$ showed no conflict of interest (clearly declaring manufacturer contribution to the manuscript) $[38,41,44,58,59,61,66,69,74], 28$ studies $(60 \%)$ had a potential conflict of interest (product supplied by a private laboratory and unclear manufacturer contribution to the manuscript) $[14,30,31,33-37,40,42,43,45-52,56,63,64,67,68,70-72,75]$, and the other 9 studies had an existing conflict of interest (either declared conflict of interest or clear existing relationship with the manufacturer) $[29,32,39,53-55,60,65,73]$.

\subsection{Reporting of Perinatal Outcomes}

A summary of the studies included in the systematic review and in the meta-analysis based on the reporting of perinatal outcomes is presented in Table 2. Detailed data on the perinatal outcomes reported in the included studies are presented in Supplementary 3: Table S2. Finally, cases of miscarriage and stillbirth were not meta-analyzed due to a lack of consensus on the outcome definition across the studies.

Table 2. Summary of included studies bases on the reporting of perinatal outcomes.

\begin{tabular}{|c|c|c|c|c|c|c|c|}
\hline \multicolumn{4}{|c|}{ Systematic Review } & \multicolumn{4}{|c|}{ Meta-Analysis } \\
\hline $\begin{array}{c}\text { Reported Perinatal } \\
\text { Outcome }\end{array}$ & $\begin{array}{c}\text { Number of } \\
\text { Reviewed Studies }\end{array}$ & $n$ & Intervention & $\begin{array}{c}\text { Number of Pooled } \\
\text { Studies }\end{array}$ & $n$ & Pooled RR/MD (95\% CI) & $\mathbf{I}^{2}$ \\
\hline \multirow[t]{2}{*}{ Preterm Birth } & 19 & 4903 & Probiotic & 11 & 2934 & $\begin{array}{l}\mathrm{RR}=1.16 \\
(0.78-1.71)\end{array}$ & $16 \%$ \\
\hline & 2 & 155 & Synbiotic & 0 & - & - & - \\
\hline \multirow[t]{2}{*}{ Weeks of Gestation } & 23 & 4144 & Probiotic & 13 & 2074 & $\begin{array}{c}\mathrm{MD}=0.03 \\
(-0.21-0.27)\end{array}$ & $78 \%$ \\
\hline & 1 & 60 & Synbiotic & 0 & - & - & - \\
\hline \multirow[t]{2}{*}{ Birth Weight } & 28 & 6666 & Probiotic & 13 & 3578 & $\begin{array}{c}\mathrm{MD}=-5.57 \\
(-38.48-27.34)\end{array}$ & $0 \%$ \\
\hline & 1 & 60 & Synbiotic & 0 & - & - & - \\
\hline \multirow{2}{*}{ Low Birth Weight } & 1 & 433 & Probiotic & 0 & - & - & - \\
\hline & 0 & - & Synbiotic & 0 & - & - & - \\
\hline \multirow{2}{*}{ Macrosomia } & 10 & 1654 & Probiotic & 3 & 94 & $\begin{array}{c}\mathrm{RR}=0.84 \\
(0.30-2.34)\end{array}$ & $60 \%$ \\
\hline & 1 & 60 & Synbiotic & 0 & - & - & - \\
\hline \multirow{2}{*}{ SGA } & 5 & 1301 & Probiotic & 0 & - & - & - \\
\hline & 0 & - & Synbiotic & 0 & - & - & - \\
\hline \multirow[t]{2}{*}{ LGA } & 7 & 1436 & Probiotic & 2 & 316 & $\begin{array}{l}\mathrm{RR}=0.98 \\
(0.60-1.61)\end{array}$ & $0 \%$ \\
\hline & 0 & - & Synbiotic & 0 & - & - & - \\
\hline \multirow[t]{2}{*}{ Cesarean Section } & 26 & 5952 & Probiotic & 17 & 3445 & $\begin{array}{l}\mathrm{RR}=0.93 \\
(0.83-1.04)\end{array}$ & $0 \%$ \\
\hline & 1 & 60 & Synbiotic & 0 & - & - & - \\
\hline \multirow{2}{*}{$\begin{array}{l}\text { Miscarriage or } \\
\text { Stillbirth }\end{array}$} & 11 & 2595 & Probiotic & 0 & - & - & - \\
\hline & 0 & - & Synbiotic & 0 & - & - & - \\
\hline
\end{tabular}

LGA: Large for Gestational Age; MD: Mean Difference; RR: Relative Risk; SGA: Small for Gestational Age. 
As presented in Supplementary 3: Table S2, the reporting of perinatal outcomes was completely heterogeneous across the studies. Only two authors described, in detail, the perinatal outcomes in the cohort of study reporting data on all of the following items: Cases of miscarriage/stillbirth, weeks of gestation, cases of preterm birth, birth weight, cases of macrosomia or LGA, cases of low birth weight or SGA, and cesarean section $[38,69]$. On the other hand, eight studies did not report data on any of the aforementioned items [30,33,35,40,42,47,67,72]. Regarding the main outcomes of the present review, the most frequently reported outcome across the studies was birth weight (29 studies, $63 \%$ ), followed by cesarean section ( 27 studies, $59 \%$ ), weeks of gestation ( 24 studies, $52 \%$ ), preterm birth (<37 weeks of gestation, 21 studies, $46 \%$ ), miscarriage/stillbirth (11 studies, $24 \%$ ), macrosomia (10 studies, 22\%), LGA (7 studies, 15\%), SGA (5 studies, $11 \%$ ), and low birth weight (1 study, $2 \%$ ). Other outcomes reported in the studies consisted of neonatal hospitalization or admission to neonatal intensive care unit (NICU) (15 studies, $33 \%$ ) $[29,34,36-38,45,48,51,58,59,66,69-71,74]$, anthropometric measures at birth (i.e., head circumference or birth length) (15 studies) $[14,29,34,36,48-51,56,59,60,66,70,73,74]$, Apgar test score at 1 or 5 minutes (11 studies, 24\%) [14,45,48,51,56,59,60,69,71,74,75], malformation/fetal abnormalities (7 studies, $15 \%$ ) [34,38,44,55,58,59,69], hypoglycemia (6 studies, $13 \%)[36,38,48,51,52,69]$, hyperbilirubinemia or jaundice (5 studies, $11 \%$ ) $[34,36,38,48,51]$, induction of labor (3 studies, $7 \%$ ) [44,59,75], polyhydramnios (3 studies) [36,48,51], cord blood pH (2 studies, $4 \%$ ) [69,75], preterm birth ( $<35$ weeks of gestation, 2 studies) [46,64], preterm birth ( $<34$ weeks of gestation, 1 study, $2 \%)$ [38], and umbilical artery resistance (1 study) [39].

\subsection{Administration of Probiotics during Pregnancy and Length of Gestation}

Eleven studies evaluated the administration of probiotic preparations to pregnant women without previous pathologies reporting the cases of preterm delivery $(<37$ weeks of gestation) in the groups analyzed. The random effects meta-analysis of these studies showed that probiotic administration during pregnancy did not have a statistically significant impact on the odds of preterm birth in the groups of study ( $R R=1.19,95 \%$ $\mathrm{CI}=0.81-1.74)$. Heterogeneity across the studies was low $\left(\mathrm{I}^{2}=14 \%\right.$; Figure 2$)$. Visual inspection of the funnel plot did not reveal potential publication bias (Figure 3).

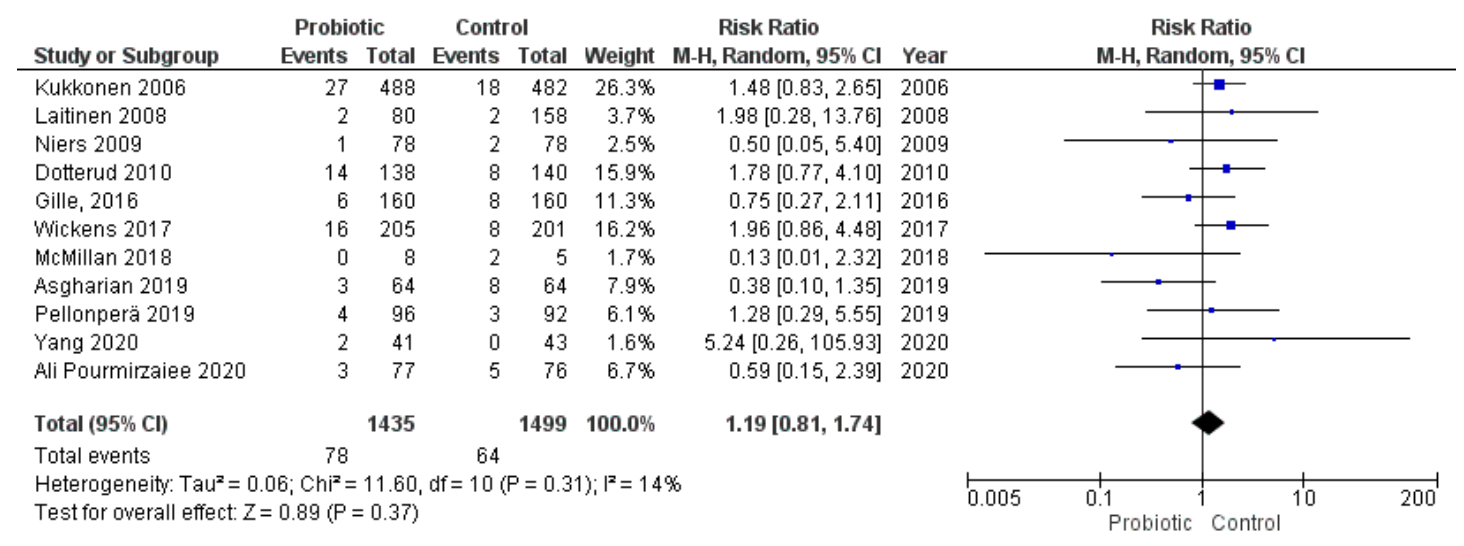

Figure 2. Meta-analysis of studies evaluating the administration of probiotic and preterm birth. 


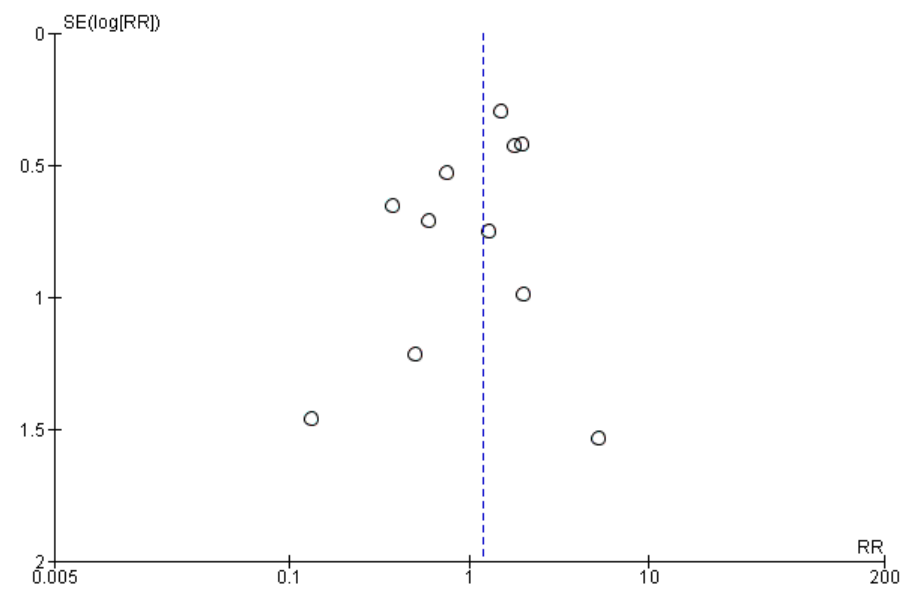

Figure 3. Funnel plot of studies evaluating the administration of probiotic and preterm birth.

We conducted a subgroup analysis excluding the trials that provided the probiotics from the last 4-6 weeks of gestation to avoid bias due to the timing of the intervention. The subgroup analysis results were similar to those obtained in the main analysis $(\mathrm{RR}=1.03$, 95\% CI = 0.53-1.99, I² = 31\%; Supplementary 4: Figure S1).

Among included studies, three authors evaluated the administration of Lactobacillus rhamnosus GR-1 in combination with Lactobacillus reuteri RC-14 and provided data on the odds of PTB. The subgroup analysis of these studies did not show a significant association between the administration of these strains during pregnancy and preterm birth $(\mathrm{RR}=0.76$, 95\% CI = 0.17-3.35, I² = 34\%; Supplementary 4: Figure S2).

Thirteen trials reported the length of gestation, providing the number of weeks of gestation in both intervention and comparison groups. Data were reported as mean \pm standard deviation in eight of the studies, while the remaining five studies reported weeks of gestation as mean $+95 \%$ CI or median + range or interquartile range.

The meta-analysis of the 13 studies showed that the administration of probiotics was not associated with the number of weeks of gestation $(\mathrm{MD}=0.03,95 \% \mathrm{CI}=-0.21-0.27$ ). However, heterogeneity across the studies was considerable $\left(\mathrm{I}^{2}=78 \%\right)$ (Figure 4$)$. We did not observe publication bias from visual inspection of the funnel plot (Figure 5).

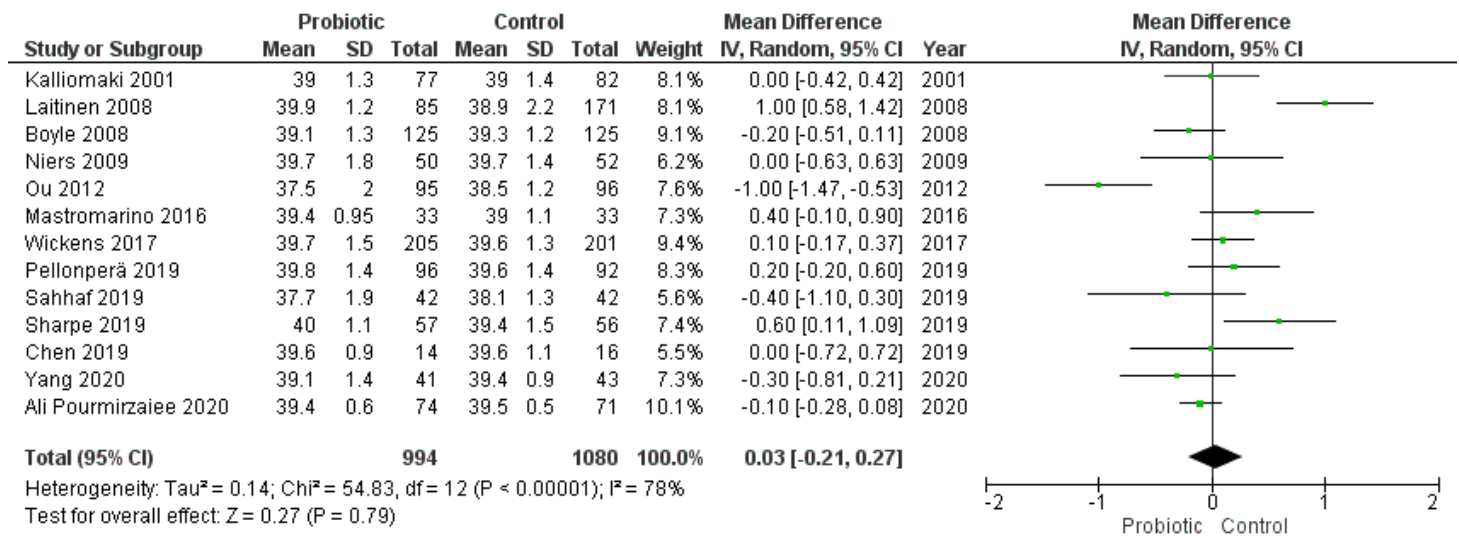

Figure 4. Meta-analysis of studies evaluating the administration of probiotic and weeks of gestation. 


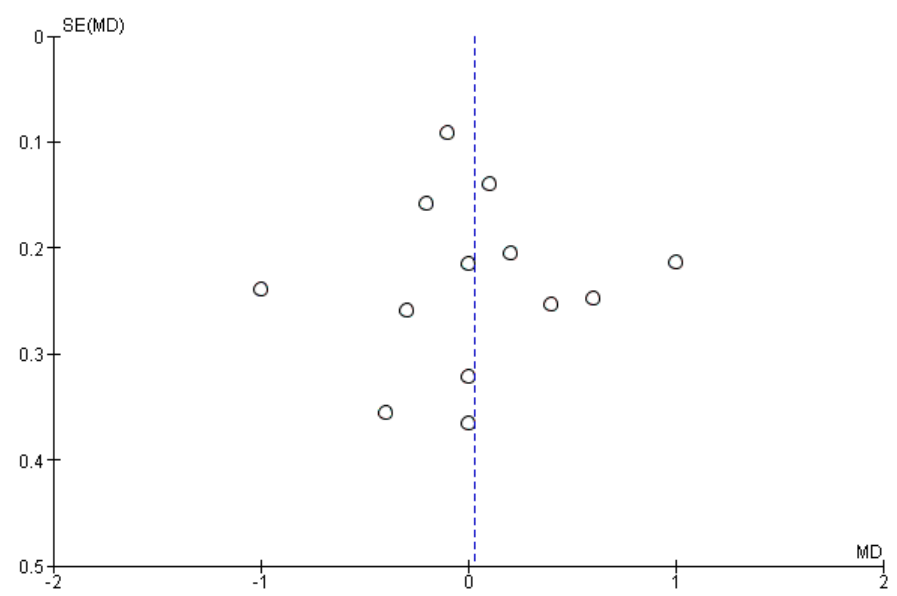

Figure 5. Funnel plot of studies evaluating the administration of probiotic and weeks of gestation.

We conducted a subgroup analysis of the eight studies that provided data on the weeks of gestation as mean \pm standard excluding the estimated values from the other five studies, yielding similar results $\left(\mathrm{MD}=0.09,95 \% \mathrm{CI}=-0.14-0.32, \mathrm{I}^{2}=56 \%\right.$; (Supplementary 4 : Figure S3).

Three of these studies specifically evaluated the administration of Lactobacillus rhamnosus GG. The subgroup analysis of these studies did not show significant changes in the pooled mean difference of the weeks of gestation $(\mathrm{MD}=-0.38,95 \% \mathrm{CI}=-0.92-0.15$, $\mathrm{I}^{2}=56 \%$; Supplementary 4: Figure S4). Similarly, the subgroup analysis of the two studies that used a combination of Lactobacillus rhamnosus GR-1 and Lactobacillus reuteri RC-14 did not significantly influence the meta-analysis results $(\mathrm{MD}=0.18,95 \% \mathrm{CI}=-0.75-1.11$, $\mathrm{I}^{2}=86 \%$; Supplementary 4: Figure S5).

\subsection{Administration of Probiotics during Pregnancy and Birth Weight}

Thirteen studies reported data on birth weight either as mean \pm standard deviation (eight studies) or as mean $+95 \%$ CI or median + range/interquartile range (five studies). The random effects meta-analysis of these studies showed that administration of probiotics during pregnancy did not have a significant impact on birth weight $(\mathrm{MD}=-5.36,95 \% \mathrm{CI}$ $=-37.60-26.89$ ) (Figure 6). Heterogeneity across included studies was low $\left(\mathrm{I}^{2}=0 \%\right)$. We did not observe publication bias after visual inspection of funnel plots (Figure 7).

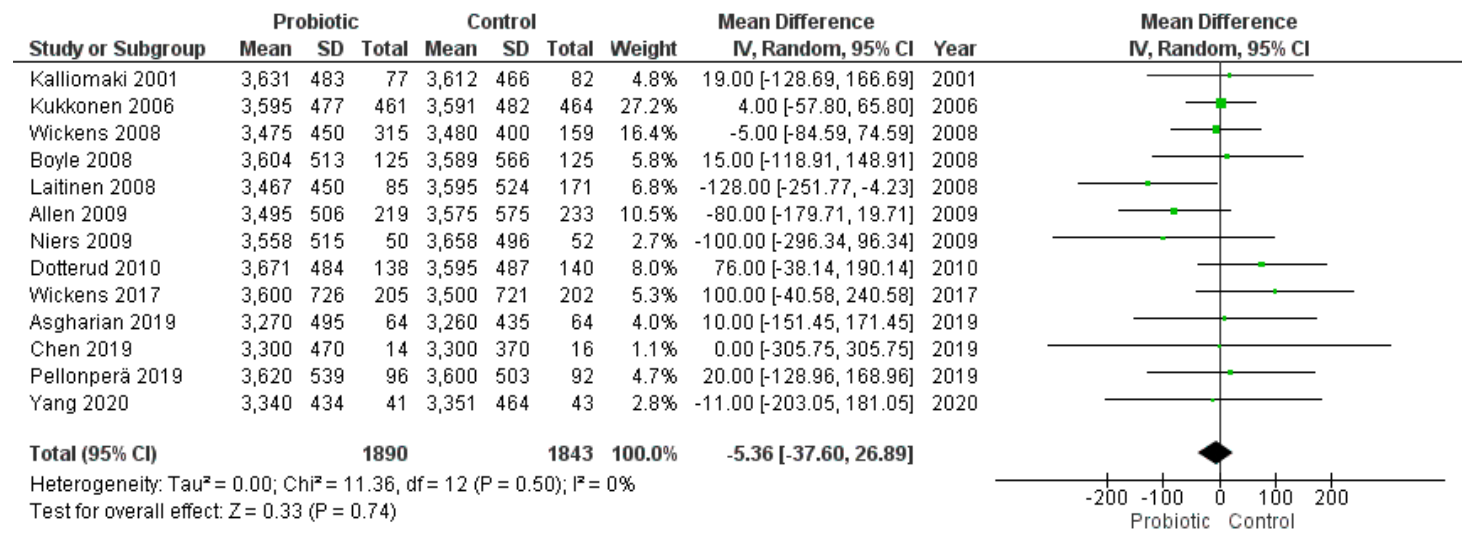

Figure 6. Meta-analysis of studies evaluating the administration of probiotic and birth weight. 


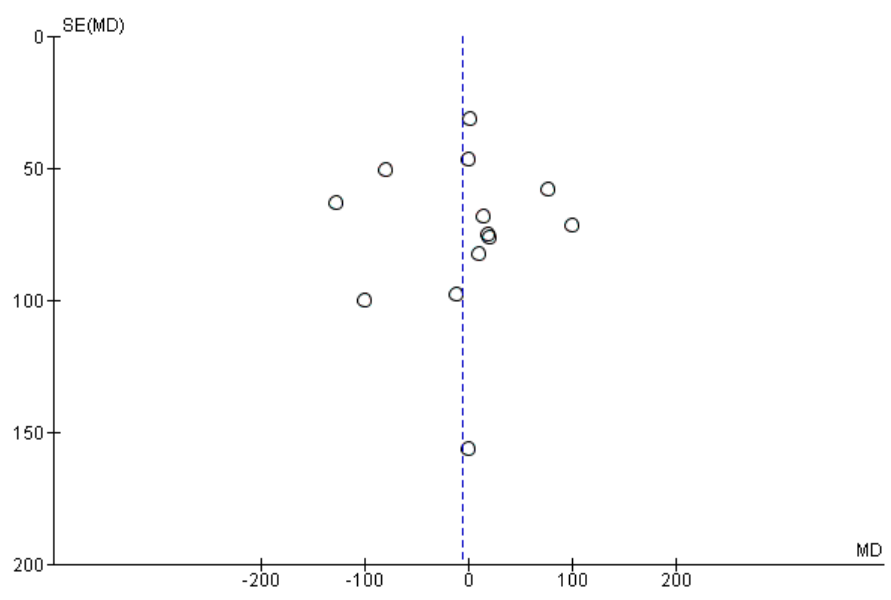

Figure 7. Funnel plot of studies evaluating the administration of probiotic and birth weight.

Subgroup analysis of only the eight studies that provided birth weight as mean \pm standard deviation did not significantly modify the meta-analysis results (MD = 10.80, 95\% CI $=-28.03-49.62, \mathrm{I}^{2}=0 \%$; Supplementary 4: Figure S6). In the same fashion, subgroup analysis excluding studies conducted during the last 4-6 weeks of gestation did not modified the meta-analysis results $\left(\mathrm{MD}=-7.43,95 \% \mathrm{CI}=-80.27-65.41 .01, \mathrm{I}^{2}=18 \%\right.$; Supplementary 4: Figure S7).

We also separately analyzed two studies that used Lactobacillus rhamnosus GG providing data on birth weight yielding similar results $(\mathrm{MD}=16.80,95 \% \mathrm{CI}=-82.40-116.01$, $\mathrm{I}^{2}=0 \%$; Supplementary 4: Figure S8).

None of the studies that provided the cases of low birth weight ( $<2500 \mathrm{~g}$ at birth) or SGA (birth weight $<10$ th percentile for the gestational age) met the inclusion criteria for the meta-analysis. On the other hand, three studies reported the cases of macrosomia (>4000 $\mathrm{g}$ at birth) in the cohort of study and two other trials reported the cases of LGA (birth weight $>90$ th percentile for the gestational age). However, Sahhaf et al. [70] did not specify the definition of macrosomia used. The meta-analysis of these studies showed that probiotic consumption during pregnancy was not associated with an increased risk of macrosomia or LGA (macrosomia: $\mathrm{RR}=0.84,95 \% \mathrm{CI}=0.30-2.34, \mathrm{I}^{2}=60 \%$; LGA: RR $=0.98$, $95 \%$ CI $=0.60-1.61, I^{2}=0 \%$; Figures 8 and 9). Exclusion of the study conducted by Sahhaf et al. [70] (which was the main cause of heterogeneity) did not significantly modify this correlation (data not shown).

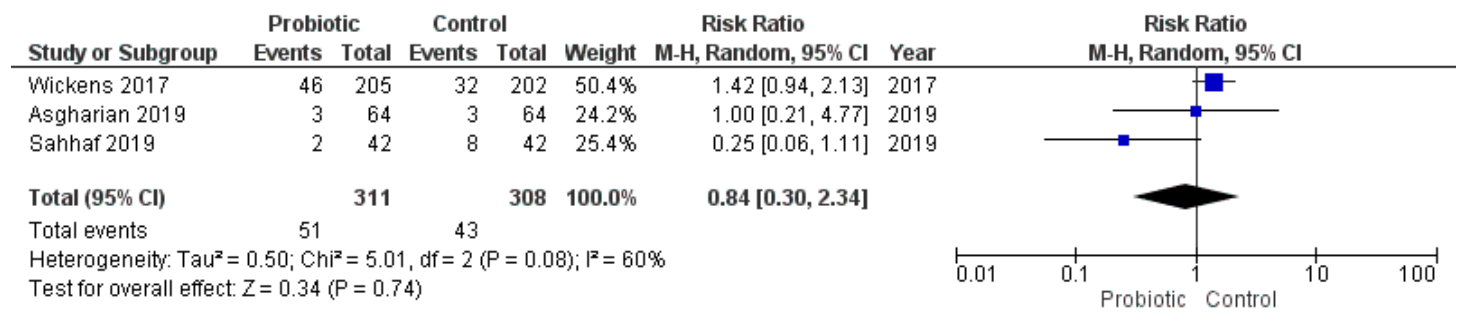

Figure 8. Meta-analysis of studies evaluating the administration of probiotic and macrosomia. 


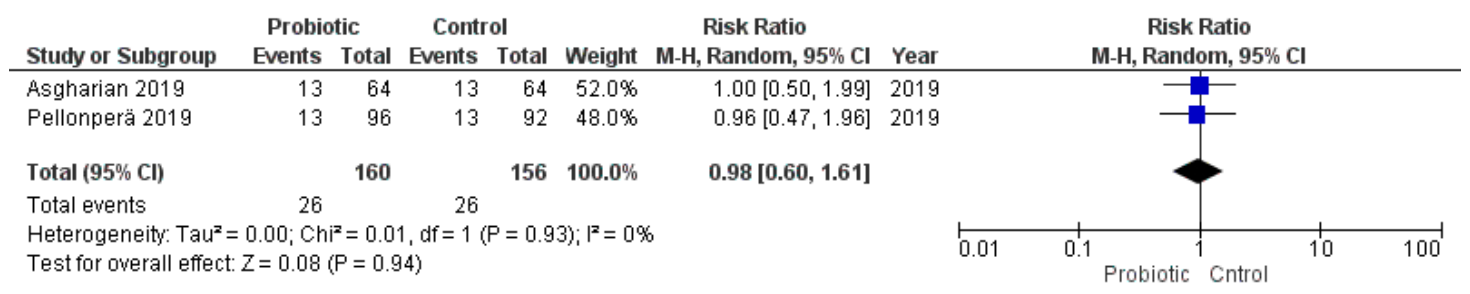

Figure 9. Meta-analysis of studies evaluating the administration of probiotic and large for gestational age.

\subsection{Administration of Probiotics during Pregnancy and Cesarean Section}

Seventeen studies reported the cases of cesarean section in the groups of study and met the inclusion criteria for the meta-analysis. The random effects meta-analysis of these studies showed that administration of probiotics during pregnancy did not influence cesarean section rate $(R R=0.98,95 \% C I=0.87-1.10$; Figure 10$)$. The studies were homogeneous $\left(\mathrm{I}^{2}=0 \%\right)$. Visual inspection of the funnel plot did not reveal publication bias (Figure 11).

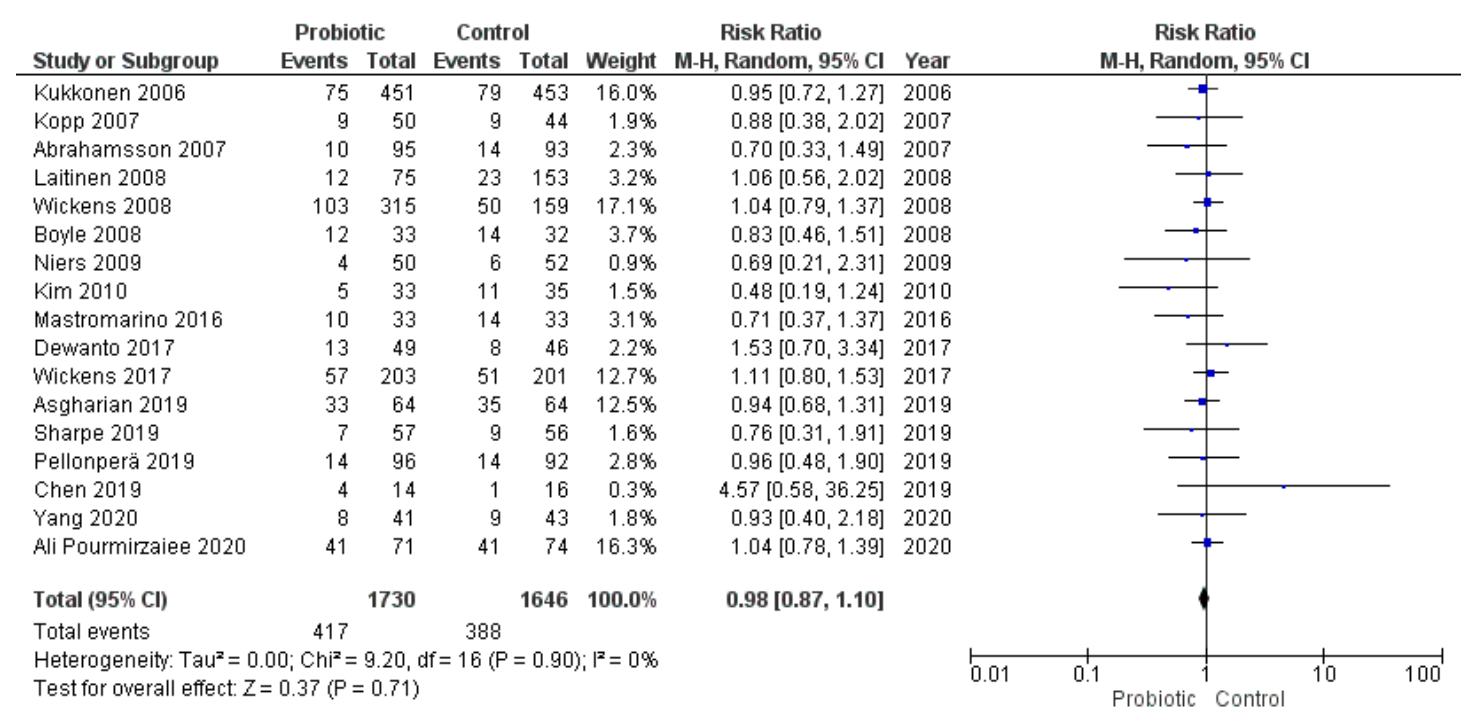

Figure 10. Meta-analysis of studies evaluating the administration of probiotic and cesarean section.

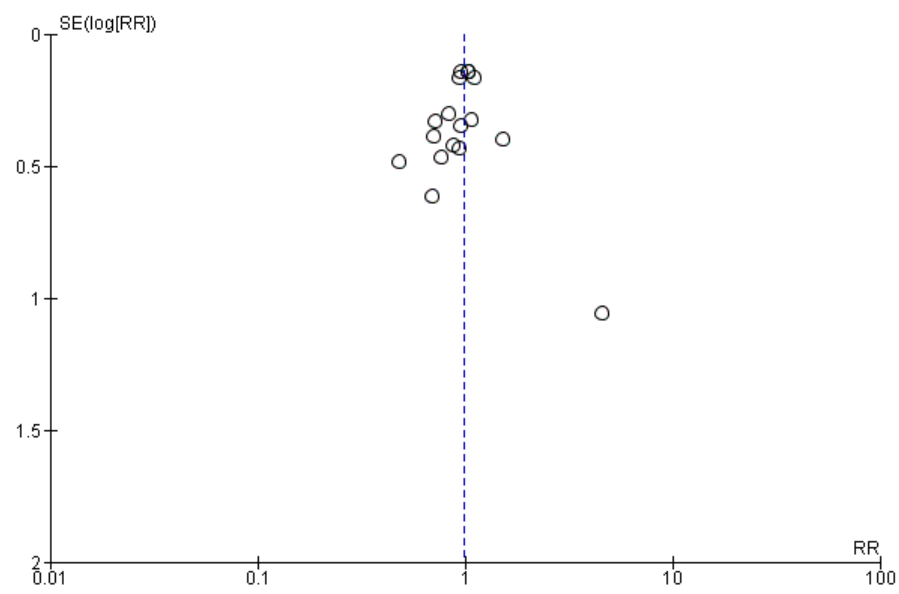

Figure 11. Funnel plot of studies evaluating the administration of probiotic and cesarean section.

Two authors evaluated the administration of L. rhamnosus GG and provided data on cesarean section cases. The subgroup analysis of these studies did not substantially modify the meta-analysis results $\left(\mathrm{RR}=0.85,95 \% \mathrm{CI}=0.52-1.38, \mathrm{I}^{2}=0 \%\right.$; Supplementary 4 : 
Figure S9). In line with this, the subgroup analysis of two other authors that administered a combination of L. rhamnosus GR-1 and L. reuteri RC-14 did not show a significant correlation with cesarean section $\left(\mathrm{RR}=0.85,95 \% \mathrm{CI}=0.46-1.59, \mathrm{I}^{2}=0 \%\right.$; Supplementary 4: Figure S10).

\section{Discussion}

The present systematic review and meta-analysis aimed at evaluating the reporting of perinatal outcomes in randomized controlled trials analyzing the effect that the administration of probiotic microorganisms might exert on these outcomes. We included 46 studies in the present review, 25 of which were meta-analyzed. The random effects meta-analysis results showed that the administration of probiotics during pregnancy was not associated with any perinatal outcome evaluated. However, the reporting of perinatal outcomes was completely heterogeneous across the included studies, and only birth weight, cases of cesarean section, and weeks of gestation were reported in more than $50 \%$ of the studies.

By definition, probiotic microorganisms, when administered in adequate amounts, confer a health benefit on the host [1,2]. Nonetheless, probiotics must also be safe for their intended use [76]. In this sense, an exhaustive review on this subject including 384 RCTs involving probiotics, prebiotics, and synbiotics concluded that the reporting of adverse events in most of these trials was lacking and inadequate [77]. Pregnant women are an immunologically vulnerable population, and the mechanisms by which probiotic microorganisms might stimulate or suppress the immune system are not clear [76]. Therefore, not only adverse events (which is out of the scope of the present review), but also perinatal outcomes should be considered when conducting probiotic trials in pregnant women.

Sample size is usually a limitation of probiotic trials when assessing differences in perinatal outcomes [78,79]. In this regard, meta-analyses help to overcome this limitation by pooling data from several studies. In line with the present research, other reviews and meta-analyses on this topic have concluded that probiotics and synbiotics do not positively or negatively influence perinatal outcomes $[10,17,18]$. Nonetheless, the authors of these reviews coincide on affirming that the reporting of these outcomes is highly heterogeneous, with some authors reporting only weeks of gestation at delivery [68] or cases of preterm labor [64] without further considering other outcomes. Furthermore, some authors have reported perinatal outcomes in secondary analyses even when they were not provided in the original research $[57,62]$. This could imply selective report bias when assessing probiotics safety.

The meta-analysis results showed that the administration of probiotics was not associated with preterm birth $(\mathrm{RR}=1.19,95 \% \mathrm{CI}=0.81-1.74)$ or birth weight $(\mathrm{MD}=-5.36$, $95 \% \mathrm{CI}=-37.60-26.89)$. The lack of association could be caused by the timing of the intervention given that several included studies administered probiotic preparations from the last 4-6 weeks of gestation to delivery or postpartum. However, the subgroup analysis excluding these studies did not yield different results. Therefore, it seems unlikely that the timing of the intervention would have a significant impact on meta-analysis conclusions. Nonetheless, it is remarkable that only 142 cases of preterm birth were included in the meta-analysis from a total of 2934 participants, which implies a low rate of preterm delivery in these studies analyzed (4.8\%) compared to global estimates (10.6\%) [80]. Similar rates of preterm birth were observed in another meta-analysis [10]. Given the low reported incidence of preterm birth in the studies analyzed, future studies with a bigger sample size are required to evaluate the effects of probiotics on this outcome.

Birth weight was mostly reported as a continuous variable in the studies. In this sense, SGA and low birth weight were clearly underreported outcomes across the studies and could not be included in the meta-analysis. On the other hand, the majority of the studies reporting cases of LGA or macrosomia were conducted in pregnant women with GDM and were thus excluded from the analyses. Finally, only three studies reporting cases of macrosomia and two studies reporting LGA cases were meta-analyzed. Due to the low number of pooled studies, it is not possible to draw solid conclusions from the 
meta-analyses regarding LGA and macrosomia. More studies are necessary to evaluate the influence of probiotics administration on SGA and low birth weight.

Mounting evidence suggests that probiotics' effects are strain-dependent [19]. However, the meta-analyses conducted to date were not able to pool data from specific strains due to the heterogeneity of included studies. In the present review, we conducted subgroup analyses of studies evaluating the administration of L. rhamnosus GG, L. rhamnosus GR-1, and $L$. reuteri $\mathrm{RC}-14$. In this regard, L. rhamnosus GR-1 has been suggested to present beneficial properties for the prevention of preterm birth in animal models [81]. Our results showed that the administration of L. rhamnosus GG was not associated with higher or lower birth weight in the included studies. Similarly, neither L. rhamnosus GG nor L. rhamnosus GR-1 in combination with L. reuteri RC-14, were associated with the number of weeks of gestation of cesarean section rate. However, almost one-third of the authors did not report the probiotic strains administered, and the small number of studies included in the sub-analyses makes it impossible to draw strong conclusions from these results.

Finally, the present research is not exempt from limitations. We did not exclude studies analyzing twin pregnancies, which could bias our results given that multiple pregnancies have a higher risk of adverse perinatal outcomes. We did not ask the authors of the studies for nonreported data, which could have increased the number of studies meta-analyzed. Lastly, we did not analyze maternal outcomes (i.e., preeclampsia) or adverse events (i.e., diarrhea), which are subjects of much deeper research on probiotic safety.

\section{Conclusions}

The meta-analysis results at the genus or species level showed that the administration of probiotic microorganisms during pregnancy does not have any positive or negative impact on birth weight, length of gestation, and cesarean section. The sub-analyses at the strain level did not modify these results. However, the number of studies reporting the same perinatal outcome and evaluating the administration of the same probiotic strains was very low, and it is thus not possible to draw strong conclusions from the sub-analyses results regarding the effect that specific probiotic strains might exert on perinatal outcomes. The reporting of perinatal outcomes was inconsistent across the included studies. Future probiotic randomized controlled trials should report perinatal outcomes and probiotic strains in order to shed light on the effects that probiotic microorganisms exert on pregnancy, placing emphasis on the safety of these interventions.

Supplementary Materials: The following are available online at https:/ / www.mdpi.com/2072-664 3/13/1/256/s1, Search strategy; Table S1: Risk of Bias Assessment; Table S2: Reporting of perinatal outcomes in individual studies. Figure S1: Random effect meta-analysis of studies evaluating the administration of probiotics during early or mid-pregnancy and preterm birth, Figure S2: Random effect meta-analysis of studies evaluating the administration of L. rhamnosus GR-1 in combination with L. reuteri RC-14 and preterm birth. Figure S3: Random effect meta-analysis of studies evaluating the administration of probiotics and weeks of gestation reported as mean \pm standard deviation. Figure S4: Random effect meta-analysis of studies evaluating the administration of L. rhamnosus GG and weeks of gestation. Figure S5: Random effect meta-analysis of studies evaluating the administration of L. rhamnosus GR-1 in combination with L. reuteri RC-14 and weeks of gestation. Figure S6: Metaanalysis of studies evaluating the administration of probiotic and birth weight reported as mean \pm standard deviation. Figure S7: Random effect meta-analysis of studies evaluating the administration of probiotics during early or mid-pregnancy and birth weight. Figure S8: Random effect meta-analysis of studies evaluating the administration of L. rhamnosus GG and birth weight. Figure S9: Random effect meta-analysis of studies evaluating the administration of L. rhamnosus GG and cesarean section. Figure S10: Random effect meta-analysis of studies evaluating the administration of L. rhamnosus GR-1 in combination with L. reuteri RC-14 and cesarean section. 
Author Contributions: Conceptualization, Í.M.P.-C., A.L.-C. and M.J.A.-C.; methodology, Í.M.P.-C.; software, I.M.P.-C.; formal analysis, Í.M.P.-C. and J.L.G.-V.; investigation, Í.M.P.-C., M.J.A.-C., A.M.R.C.; writing-original draft preparation, Í.M.P.-C.; writing—review and editing, A.L.-C., M.J.A.-C., R.F.-C., A.M.R.-C. and J.L.G.-V.; M.J.A.-C.; funding acquisition, M.J.A.-C. and R.F.-C. All authors have read and agreed to the published version of the manuscript.

Funding: Research reported in this publication was funded by the Spanish Ministry of Science, Innovation, and Universities (Project FIS-ISCIII, P117/02305) and co-founded by FEDER, “investing in your future".

Institutional Review Board Statement: Not applicable.

Informed Consent Statement: Not applicable.

Data Availability Statement: Data sharing not applicable.

Acknowledgments: This paper is part of the doctoral thesis of I.M.P.-C in the Clinical Medicine and Public Health Doctoral Program of the University of Granada, Spain.

Conflicts of Interest: The authors declare no conflict of interest.

\section{References}

1. Joint FAO/WHO Expert Consultation on Evaluation of Health and Nutritional Properties of Probiotics in Food including Powder Milk with Live Lactic Acid Bacteria. In Proceedings of the Food and Agriculture Organizationof the United Nations, Amerian Córdoba Park Hotel, Córdoba, Argentina, 1-4 October 2001.

2. Hill, C.; Guarner, F.; Reid, G.; Gibson, G.R.; Merenstein, D.J.; Pot, B.; Morelli, L.; Canani, R.B.; Flint, H.J.; Salminen, S.; et al. Expert consensus document: The international scientific association for probiotics and prebiotics consensus statement on the scope and appropriate use of the term probiotic. Nat. Rev. Gastroenterol. Hepatol. 2014, 11, 506-514. [CrossRef]

3. Swanson, K.S.; Gibson, G.R.; Hutkins, R.; Reimer, R.A.; Reid, G.; Verbeke, K.; Scott, K.P.; Holscher, H.D.; Azad, M.B.; Delzenne, N.M.; et al. The International Scientific Association for Probiotics and Prebiotics (ISAPP) consensus statement on the definition and scope of synbiotics. Nat. Rev. Gastroenterol. Hepatol. 2020, 17, 687-701. [CrossRef] [PubMed]

4. de Simone, C. The unregulated probiotic market. Clin. Gastroenterol. Hepatol. 2019, 17, 809-817. [CrossRef] [PubMed]

5. Probiotics Market Size \& Share I Industry Research Report. 2019-2025. Available online: https:/ /www.grandviewresearch.com/ industry-analysis / probiotics-market (accessed on 6 December 2020).

6. Goldin, B.R.; Gorbach, S.L. Clinical indications for probiotics: An overview. Clin. Infect. Dis. 2008, 46 (Suppl. S2). [CrossRef] [PubMed]

7. Łagowska, K.; Malinowska, A.M.; Zawieja, B.; Zawieja, E. Improvement of glucose metabolism in pregnant women through probiotic supplementation depends on gestational diabetes status: Meta-Analysis. Sci. Rep. 2020, 10. [CrossRef]

8. Kuang, L.; Jiang, Y. Effect of probiotic supplementation in pregnant women: A meta-analysis of randomised controlled trials. Br. J. Nutr. 2020, 123, 870-880. [CrossRef] [PubMed]

9. Zhang, J.; Ma, S.; Wu, S.; Guo, C.; Long, S.; Tan, H. Effects of probiotic supplement in pregnant women with gestational diabetes mellitus: A systematic review and meta-analysis of randomized controlled trials. J. Diabetes Res. 2019, 2019, 5364730. [CrossRef]

10. Jarde, A.; Lewis-Mikhael, A.-M.; Moayyedi, P.; Stearns, J.C.; Collins, S.M.; Beyene, J.; McDonald, S.D. Pregnancy outcomes in women taking probiotics or prebiotics: A systematic review and meta-analysis. BMC Pregnancy Childbirth 2018, 18. [CrossRef]

11. Mor, G.; Aldo, P.; Alvero, A.B. The unique immunological and microbial aspects of pregnancy. Nat. Rev. Immunol. 2017, 17, 469-482. [CrossRef]

12. Bastek, J.A.; Gómez, L.M.; Elovitz, M.A. The role of inflammation and infection in preterm birth. Clin. Perinatol. 2011, 38, 385-406. [CrossRef]

13. Ashraf, R.; Shah, N.P. Immune system stimulation by probiotic microorganisms. Crit. Rev. Food Sci. Nutr. 2014, 54, 938-956. [CrossRef] [PubMed]

14. Chen, Y.; Li, Z.; Tye, K.D.; Luo, H.; Tang, X.; Liao, Y.; Wang, D.; Zhou, J.; Yang, P.; Li, Y.; et al. Probiotic supplementation during human pregnancy affects the gut microbiota and immune status. Front. Cell. Infect. Microbiol. 2019, 9, 254. [CrossRef] [PubMed]

15. Forsberg, A.; Abrahamsson, T.R.; Nilsson, L.; Ernerudh, J.; Duchén, K.; Jenmalm, M.C. Changes in peripheral immune populations during pregnancy and modulation by probiotics and $\omega-3$ fatty acids. Sci. Rep. 2020, 10. [CrossRef] [PubMed]

16. Baldassarre, M.E.; Palladino, V.; Amoruso, A.; Pindinelli, S.; Mastromarino, P.; Fanelli, M.; Di Mauro, A.; Laforgia, N. Rationale of probiotic supplementation during pregnancy and neonatal period. Nutrients 2018, 10, 1693. [CrossRef] [PubMed]

17. Dugoua, J.J.; Machado, M.; Zhu, X.; Chen, X.; Koren, G.; Einarson, T.R. Probiotic safety in pregnancy: A systematic review and meta-analysis of randomized controlled trials of lactobacillus, bifidobacterium, and saccharomyces spp. J. Obstet. Gynaecol. Can. 2009, 31, 542-552. [CrossRef]

18. Vandevusse, L.; Hanson, L.; Safdar, N. Perinatal outcomes of prenatal probiotic and prebiotic administration: An integrative review. J. Perinat. Neonatal Nurs. 2013, 27, 288-301. [CrossRef] [PubMed] 
19. McFarland, L.V.; Evans, C.T.; Goldstein, E.J.C. Strain-Specificity and disease-specificity of probiotic efficacy: A systematic review and meta-analysis. Front. Med. 2018, 5. [CrossRef]

20. Lerner, A.; Shoenfeld, Y.; Matthias, T. Probiotics: If it does not help it does not do any harm. Really? Microorganisms 2019, 7, 104. [CrossRef]

21. Moher, D.; Liberati, A.; Tetzlaff, J.; Altman, D.G.; The PRISMA Group. Preferred reporting items for systematic reviews and meta-analyses: The PRISMA statement. PLoS Med. 2009, 6. [CrossRef]

22. World Health Organization. WHO: Recommended definitions, terminology and format for statistical tables related to the perinatal period and use of a new certificate for cause of perinatal deaths. Acta Obstet. Gynecol. Scand. 1977, 56, 247-253.

23. Battaglia, F.C.; Lubchenco, L.O. A practical classification of newborn infants by weight and gestational age. J. Pediatrics 1967, 71 , 159-163. [CrossRef]

24. World Health Organization. ICD-10: International Statistical Classification of Diseases and Related Health Problems: Tenth Revision, 2nd ed.; World Health Organization: Geneva, Switzerland, 2004.

25. ACOG. Clinical management guidelines for obstetrician-Gynecologists. Obstet. Gynecol. 2019, 133, $168-186$.

26. Higgins, J.P.; Savović, J.; Page, M.J.; Elbers, R.G.; Sterne, J.A. Assessing risk of bias in a randomized trial. Cochrane Handb. Syst. Rev. Interv. 2019, 205-228. [CrossRef]

27. Cochrane Handbook for Systematic Reviews of Interventions | Cochrane Training. Available online: https://training.cochrane. org/handbook/current (accessed on 6 December 2020).

28. Wan, X.; Wang, W.; Liu, J.; Tong, T. Estimating the sample mean and standard deviation from the sample size, median, range and/or interquartile range. BMC Med. Res. Methodol. 2014, 14. [CrossRef] [PubMed]

29. Abrahamsson, T.R.; Jakobsson, T.; Böttcher, M.F.; Fredrikson, M.; Jenmalm, M.C.; Björkstén, B.; Oldaeus, G. Probiotics in prevention of IgE-associated eczema: A double-blind, randomized, placebo-controlled trial. J. Allergy Clin. Immunol. 2007, 119, 1174-1180. [CrossRef] [PubMed]

30. Ahmadi, S.; Jamilian, M.; Tajabadi-Ebrahimi, M.; Jafari, P.; Asemi, Z. The effects of synbiotic supplementation on markers of insulin metabolism and lipid profiles in gestational diabetes: A randomised, double-blind, placebo-controlled trial. Br. J. Nutr. 2016, 116, 1394-1401. [CrossRef]

31. Pourmirzaiee, M.A.; Famouri, F.; Moazeni, W.; Hassanzadeh, A.; Hajihashemi, M. The efficacy of the prenatal administration of lactobacillus reuteri LR92 DSM 26866 on the prevention of infantile colic: A randomized control trial. Eur. J. Pediatr. 2020, 179, 1619-1626. [CrossRef]

32. Allen, S.J.; Jordan, S.; Storey, M.; Thornton, C.A.; Gravenor, M.; Garaiova, I.; Plummer, S.F.; Wang, D.; Morgan, G. Dietary supplementation with lactobacilli and bifidobacteria is well tolerated and not associated with adverse events during late pregnancy and early infancy. J. Nutr. 2010, 140, 483-488. [CrossRef]

33. Asemi, Z.; Jazayeri, S.; Najafi, M.; Samimi, M.; Mofid, V.; Shidfar, F.; Shakeri, H.; Esmaillzadeh, A. Effect of daily consumption of probiotic yogurt on oxidative stress in pregnant women: A randomized controlled clinical trial. Ann. Nutr. Metab. 2012, 60, 62-68. [CrossRef]

34. Asgharian, H.; Homayouni-Rad, A.; Mirghafourvand, M.; Mohammad-Alizadeh-Charandabi, S. Effect of probiotic yoghurt on plasma glucose in overweight and obese pregnant women: A randomized controlled clinical trial. Eur. J. Nutr. 2020, 59, 205-215. [CrossRef]

35. Babadi, M.; Khorshidi, A.; Aghadavood, E.; Samimi, M.; Kavossian, E.; Bahmani, F.; Mafi, A.; Shafabakhsh, R.; Satari, M.; Asemi, Z. The effects of probiotic supplementation on genetic and metabolic profiles in patients with gestational diabetes mellitus: A randomized, double-blind, placebo-controlled trial. Probiotics Antimicrob. Proteins 2019, 11, 1227-1235. [CrossRef] [PubMed]

36. Badehnoosh, B.; Karamali, M.; Zarrati, M.; Jamilian, M.; Bahmani, F.; Tajabadi-Ebrahimi, M.; Jafari, P.; Rahmani, E.; Asemi, Z. The effects of probiotic supplementation on biomarkers of inflammation, oxidative stress and pregnancy outcomes in gestational diabetes. J. Matern. Neonatal Med. 2018, 31, 1128-1136. [CrossRef] [PubMed]

37. Boyle, R.J.; Ismail, I.H.; Kivivuori, S.; Licciardi, P.V.; Robins-Browne, R.M.; Mah, L.-J.; Axelrad, C.; Moore, S.; Donath, S.; Carlin, J.B.; et al. Lactobacillus GG treatment during pregnancy for the prevention of eczema: A randomized controlled trial. Allergy 2011, 66, 509-516. [CrossRef] [PubMed]

38. Callaway, L.K.; McIntyre, H.D.; Barrett, H.L.; Foxcroft, K.; Tremellen, A.; Lingwood, B.E.; Tobin, J.M.; Wilkinson, S.; Kothari, A.; Morrison, M.; et al. Probiotics for the prevention of gestational diabetes mellitus in overweight and obese women: Findings from the SPRING double-blind randomized controlled trial. Diabetes Care 2019, 42, 364-371. [CrossRef] [PubMed]

39. Dewanto, N.E.F.; Firmansyah, A.; Sungkar, A.; Dharmasetiawani, N.; Kresno, S.B.; Suradi, R.; Bardosono, S.; Prasetyo, D. The effect of bifidobacterium animalis lactis HNO19 supplementation among pregnant and lactating women on interleukin-8 level in breast milk and infant's gut mucosal integrity. Med J. Indones. 2017, 26, 204-211. [CrossRef]

40. Dolatkhah, N.; Hajifaraji, M.; Abbasalizadeh, F.; Aghamohammadzadeh, N.; Mehrabi, Y.; Abbasi, M.M. Is there a value for probiotic supplements in gestational diabetes mellitus? A randomized clinical trial. J. Health Popul. Nutr. 2015, 33, 25. [CrossRef] [PubMed]

41. Dotterud, C.K.; Storrø, O.; Johnsen, R.; Oien, T. Probiotics in pregnant women to prevent allergic disease: A randomized, double-blind trial. Br. J. Dermatol. 2010, 163, 616-623. [CrossRef]

42. Fernández, L.; Cárdenas, N.; Arroyo, R.; Manzano, S.; Jiménez, E.; Martín, V.; Rodríguez, J.M. Prevention of infectious mastitis by oral administration of lactobacillus salivarius PS2 during late pregnancy. Clin. Infect. Dis. 2016, 62, 568-573. [CrossRef] 
43. Gille, C.; Böer, B.; Marschal, M.; Urschitz, M.S.; Heinecke, V.; Hund, V.; Speidel, S.; Tarnow, I.; Mylonas, I.; Franz, A.; et al. Effect of probiotics on vaginal health in pregnancy. EFFPRO, a randomized controlled trial. Am. J. Obstet. Gynecol. 2016, 215, 608.e1-608.e7. [CrossRef]

44. Halkjær, S.I.; de Knegt, V.E.; Lo, B.; Nilas, L.; Cortes, D.; Pedersen, A.E.; Mirsepasi-Lauridsen, H.C.; Andersen, L.O.; Nielsen, H.V.; Stensvold, C.R.; et al. Multistrain probiotic increases the gut microbiota diversity in obese pregnant women: Results from a randomized, double-blind placebo-controlled study. Curr. Dev. Nutr. 2020, 4, nzaa095. [CrossRef]

45. Ho, M.; Chang, Y.-Y.; Chang, W.-C.; Lin, H.-C.; Wang, M.-H.; Lin, W.-C.; Chiu, T.-H. Oral lactobacillus rhamnosus GR-1 and lactobacillus reuteri RC-14 to reduce group B streptococcus colonization in pregnant women: A randomized controlled trial. Taiwan. J. Obstet. Gynecol. 2016, 55, 515-518. [CrossRef] [PubMed]

46. Jafarnejad, S.; Saremi, S.; Jafarnejad, F.; Arab, A. Effects of a multispecies probiotic mixture on glycemic control and inflammatory status in women with gestational diabetes: A randomized controlled clinical trial. J. Nutr. Metab. 2016, 2016. [CrossRef] [PubMed]

47. Jamilian, M.; Bahmani, F.; Vahedpoor, Z.; Salmani, A.; Tajabadi-Ebrahimi, M.; Jafari, P.; Hashemi Dizaji, S.; Asemi, Z. Effects of probiotic supplementation on metabolic status in pregnant women: A randomized, double-blind, placebo-controlled trial. Arch Iran. Med. 2016, 19, 687-692. [PubMed]

48. Jamilian, M.; Amirani, E.; Asemi, Z. The effects of vitamin D and probiotic co-supplementation on glucose homeostasis, inflammation, oxidative stress and pregnancy outcomes in gestational diabetes: A randomized, double-blind, placebo-controlled trial. Clin. Nutr. 2019, 38, 2098-2105. [CrossRef] [PubMed]

49. Kalliomäki, M.; Salminen, S.; Arvilommi, H.; Kero, P.; Koskinen, P.; Isolauri, E. Probiotics in primary prevention of atopic disease: A randomised placebo-controlled trial. Lancet 2001, 357, 1076-1079. [CrossRef]

50. Karamali, M.; Dadkhah, F.; Sadrkhanlou, M.; Jamilian, M.; Ahmadi, S.; Tajabadi-Ebrahimi, M.; Jafari, P.; Asemi, Z. Effects of probiotic supplementation on glycaemic control and lipid profiles in gestational diabetes: A randomized, double-blind, placebo-controlled trial. Diabetes Metab. 2016, 42, 234-241. [CrossRef]

51. Karamali, M.; Nasiri, N.; Shavazi, N.T.; Jamilian, M.; Bahmani, F.; Tajabadi-Ebrahimi, M.; Asemi, Z. The effects of synbiotic supplementation on pregnancy outcomes in gestational diabetes. Probiotics Antimicrob. Proteins 2018, 10, 496-503. [CrossRef]

52. Kijmanawat, A.; Panburana, P.; Reutrakul, S.; Tangshewinsirikul, C. Effects of probiotic supplements on insulin resistance in gestational diabetes mellitus: A double-blind randomized controlled trial. J. Diabetes Investig. 2019, 10, 163-170. [CrossRef]

53. Kim, J.Y.; Kwon, J.H.; Ahn, S.H.; Lee, S.I.; Han, Y.S.; Choi, Y.O.; Lee, S.Y.; Ahn, K.M.; Ji, G.E. Effect of probiotic mix (Bifidobacterium bifidum, Bifidobacterium lactis, Lactobacillus acidophilus) in the primary prevention of eczema: A double-blind, randomized, placebo-controlled trial. Pediatr. Allergy Immunol. 2010, 21, e386-e393. [CrossRef]

54. Kopp, M.V.; Hennemuth, I.; Heinzmann, A.; Urbanek, R. Randomized, double-blind, placebo-controlled trial of probiotics for primary prevention: No clinical effects of lactobacillus Gg supplementation. Pediatrics 2008, 121, e850-e856. [CrossRef]

55. Kukkonen, K.; Savilahti, E.; Haahtela, T.; Juntunen-Backman, K.; Korpela, R.; Poussa, T.; Tuure, T.; Kuitunen, M. Probiotics and prebiotic galacto-oligosaccharides in the prevention of allergic diseases: A randomized, double-blind, placebo-controlled trial. J. Allergy Clin. Immunol. 2007, 119, 192-198. [CrossRef] [PubMed]

56. Laitinen, K.; Poussa, T.; Isolauri, E. Probiotics and dietary counselling contribute to glucose regulation during and after pregnancy: A randomised controlled trial. Br. J. Nutr. 2009, 101, 1679-1687. [CrossRef] [PubMed]

57. Luoto, R.; Laitinen, K.; Nermes, M.; Isolauri, E. Impact of maternal probiotic-supplemented dietary counselling on pregnancy outcome and prenatal and postnatal growth: A double-blind, placebo-controlled study. Br. J. Nutr. 2010, 103, 1792-1799. [CrossRef] [PubMed]

58. Lindsay, K.L.; Kennelly, M.; Culliton, M.; Smith, T.; Maguire, O.C.; Shanahan, F.; Brennan, L.; McAuliffe, F.M. Probiotics in obese pregnancy do not reduce maternal fasting glucose: A double-blind, placebo-controlled, randomized trial (probiotics in pregnancy study). Am. J. Clin. Nutr. 2014, 99, 1432-1439. [CrossRef]

59. Lindsay, K.L.; Brennan, L.; Kennelly, M.A.; Maguire, O.C.; Smith, T.; Curran, S.; Coffey, M.; Foley, M.E.; Hatunic, M.; Shanahan, F.; et al. Impact of probiotics in women with gestational diabetes mellitus on metabolic health: A randomized controlled trial. Am. J. Obstet. Gynecol. 2015, 212, 496.e1-496.e11. [CrossRef]

60. Mantaring, J.; Benyacoub, J.; Destura, R.; Pecquet, S.; Vidal, K.; Volger, S.; Guinto, V. Effect of maternal supplement beverage with and without probiotics during pregnancy and lactation on maternal and infant health: A randomized controlled trial in the philippines. BMC Pregnancy Childbirth 2018, 18. [CrossRef]

61. Baldassarre, M.E.; Di Mauro, A.; Mastromarino, P.; Fanelli, M.; Martinelli, D.; Urbano, F.; Capobianco, D.; Laforgia, N. Administration of a multi-strain probiotic product to women in the perinatal period differentially affects the breast milk cytokine profile and may have beneficial effects on neonatal gastrointestinal functional symptoms. A randomized clinical trial. Nutrients 2016, 8 , 677. [CrossRef]

62. Mastromarino, P.; Capobianco, D.; Miccheli, A.; Praticò, G.; Campagna, G.; Laforgia, N.; Capursi, T.; Baldassarre, M.E. Administration of a multistrain probiotic product (VSL\#3) to women in the perinatal period differentially affects breast milk beneficial microbiota in relation to mode of delivery. Pharmacol. Res. 2015, 95-96, 63-70. [CrossRef]

63. McMillan, A.; Rulisa, S.; Gloor, G.B.; Macklaim, J.M.; Sumarah, M.; Reid, G. Pilot assessment of probiotics for pregnant women in rwanda. PLoS ONE 2018, 13. [CrossRef] 
64. Nabhani, Z.; Hezaveh, S.J.G.; Razmpoosh, E.; Asghari-Jafarabadi, M.; Gargari, B.P. The effects of synbiotic supplementation on insulin resistance/sensitivity, lipid profile and total antioxidant capacity in women with gestational diabetes mellitus: A randomized double blind placebo controlled clinical trial. Diabetes Res. Clin. Pract. 2018, 138, 149-157. [CrossRef]

65. Niers, L.; Martín, R.; Rijkers, G.; Sengers, F.; Timmerman, H.; Van Uden, N.; Smidt, H.; Kimpen, J.; Hoekstra, M. The effects of selected probiotic strains on the development of eczema (the PandA Study). Allergy Eur. J. Allergy Clin. Immunol. 2009, 64, 1349-1358. [CrossRef] [PubMed]

66. Okesene-Gafa, K.A.M.; Li, M.; McKinlay, C.J.D.; Taylor, R.S.; Rush, E.C.; Wall, C.R.; Wilson, J.; Murphy, R.; Taylor, R.; Thompson, J.M.D.; et al. Effect of antenatal dietary interventions in maternal obesity on pregnancy weight-gain and birthweight: Healthy mums and babies (HUMBA) randomized trial. Am. J. Obstet. Gynecol. 2019, 221, 152.e1-152.e13. [CrossRef] [PubMed]

67. Olsen, P.; Williamson, M.; Traynor, V.; Georgiou, C. The impact of oral probiotics on vaginal group B streptococcal colonisation rates in pregnant women: A pilot randomised control study. Women Birth 2018, 31, 31-37. [CrossRef] [PubMed]

68. Ou, C.Y.; Kuo, H.C.; Wang, L.; Hsu, T.Y.; Chuang, H.; Liu, C.A.; Chang, J.C.; Yu, H.R.; Yang, K.D. Prenatal and postnatal probiotics reduces maternal but not childhood allergic diseases: A randomized, double-blind, placebo-controlled trial. Clin. Exp. Allergy 2012, 42, 1386-1396. [CrossRef] [PubMed]

69. Pellonperä, O.; Mokkala, K.; Houttu, N.; Vahlberg, T.; Koivuniemi, E.; Tertti, K.; Rönnemaa, T.; Laitinen, K. Efficacy of fish oil and/or probiotic intervention on the incidence of gestational diabetes mellitus in an at-risk group of overweight and obese women: A randomized, placebo-controlled, double-blind clinical trial. Diabetes Care 2019, 42, 1009-1017. [CrossRef]

70. Ebrahimi, F.S.; Homayouni Rad, A.; Mosen, M.; Abbasalizadeh, F.; Tabrizi, A.; Khalili, L. Effect of L. acidophilus and B. lactis on blood glucose in women with gestational diabetes mellitus: A randomized placebo-controlled trial. Diabetol. Metab. Syndr. 2019, 11, 1-7. [CrossRef]

71. Sharpe, M.; Shah, V.; Freire-Lizama, T.; Cates, E.C.; McGrath, K.; David, I.; Cowan, S.; Letkeman, J.; Stewart-Wilson, E. Effectiveness of oral intake of lactobacillus rhamnosus GR-1 and lactobacillus reuteri RC-14 on group B streptococcus colonization during pregnancy: A midwifery-led double-blind randomized controlled pilot trial. J. Matern. Neonatal Med. 2019, 7058. [CrossRef]

72. Taghizadeh, M.; Asemi, Z. Effects of synbiotic food consumption on glycemic status and serum Hs-CRP in pregnant women: A randomized controlled clinical trial. Hormones 2014, 13, 398-406. [CrossRef]

73. Wickens, K.; Black, P.N.; Stanley, T.V.; Mitchell, E.; Fitzharris, P.; Tannock, G.W.; Purdie, G.; Crane, J. A differential effect of 2 probiotics in the prevention of eczema and atopy: A double-blind, randomized, placebo-controlled trial. J. Allergy Clin. Immunol. 2008, 122, 788-794. [CrossRef]

74. Wickens, K.L.; Barthow, C.A.; Murphy, R.; Abels, P.R.; Maude, R.M.; Stone, P.R.; Mitchell, E.A.; Stanley, T.V.; Purdie, G.L.; Kang, J.M.; et al. Early pregnancy probiotic supplementation with lactobacillus rhamnosus HN001 may reduce the prevalence of gestational diabetes mellitus: A randomised controlled trial. Br. J. Nutr. 2017, 117, 804-813. [CrossRef]

75. Yang, S.; Reid, G.; Challis, J.R.G.; Gloor, G.B.; Asztalos, E.; Money, D.; Seney, S.; Bocking, A.D. Effect of oral probiotic lactobacillus rhamnosus GR-1 and lactobacillus reuteri RC-14 on the vaginal microbiota, cytokines and chemokines in pregnant women. Nutrients 2020, 12, 368. [CrossRef] [PubMed]

76. Sanders, M.E.; Akkermans, L.M.A.; Haller, D.; Hammerman, C.; Heimbach, J.; Hörmannsperger, G.; Huys, G.; Levy, D.D.; Lutgendorff, F.; Mack, D.; et al. Safety assessment of probiotics for human use. Gut Microbes 2010, 1, 164-185. [CrossRef] [PubMed]

77. Royal College of Obstetricians \& Gynaecologists; British Association for Sexual Health and HIV. The management of women of reproductive age attending non-genitourinary medicine settings complaining of vaginal discharge. J. Fam. Plan. Reprod. Health Care 2006, 32, 33-41. [CrossRef]

78. Lee, J.E.; Han, J.Y.; Choi, J.S.; Ahn, H.K.; Lee, S.W.; Kim, M.H.; Ryu, H.M.; Yang, J.H.; Nava-Ocampo, A.A.; Koren, G. Pregnancy outcome after exposure to the probiotic lactobacillus in early pregnancy. J. Obstet. Gynaecol. 2012, 32, 227-229. [CrossRef] [PubMed]

79. Wang, C.C.; Tung, Y.T.; Chang, H.C.; Lin, C.H.; Chen, Y.C. Effect of probiotic supplementation on newborn birth weight for mother with gestational diabetes mellitus or overweight/obesity: A systematic review and meta-analysis. Nutrients 2020, 12, 3477. [CrossRef] [PubMed]

80. Chawanpaiboon, S.; Vogel, J.P.; Moller, A.B.; Lumbiganon, P.; Petzold, M.; Hogan, D.; Landoulsi, S.; Jampathong, N.; Kongwattanakul, K.; Laopaiboon, M.; et al. Global, regional, and national estimates of levels of preterm birth in 2014: A systematic review and modelling analysis. Lancet Glob. Health 2019, 7, e37-e46. [CrossRef]

81. Kim, B.; Shynlova, O.; Lye, S. Probiotic lactobacillus rhamnosus GR-1 is a unique prophylactic agent that suppresses infectioninduced myometrial cell responses. Sci. Rep. 2019, 9, 4698. [CrossRef] 